\title{
An Efficient Threshold Dynamics Method for Topology Optimization for Fluids
}

\author{
Huangxin Chen ${ }^{1}$, Haitao Leng ${ }^{2}$, Dong Wang ${ }^{3}$ and Xiao-Ping Wang $3,4, *$ \\ ${ }^{1}$ School of Mathematical Sciences and Fujian Provincial Key Laboratory on \\ Mathematical Modeling and High Performance Scientific Computing, Xiamen \\ University, Fujian, 361005, China. \\ 2 School of Mathematical Sciences, South China Normal University, Guangzhou \\ 510631, Guangdong, China. \\ ${ }^{3}$ School of Science and Engineering, The Chinese University of Hong Kong, \\ Shenzhen, Guangdong 518172, China. \\ ${ }^{4}$ Department of Mathematics, The Hong Kong University of Science and Technology, \\ Clear Water Bay, Kowloon, Hong Kong, China.
}

Received 29 January 2021; Accepted 30 May 2021

\begin{abstract}
We propose an efficient threshold dynamics method for topology optimization for fluids modeled with the Stokes equation. The proposed algorithm is based on minimization of an objective energy function that consists of the dissipation power in the fluid and the perimeter approximated by nonlocal energy, subject to a fluid volume constraint and the incompressibility condition. We show that the minimization problem can be solved with an iterative scheme in which the Stokes equation is approximated by a Brinkman equation. The indicator functions of the fluid-solid regions are then updated according to simple convolutions followed by a thresholding step. We prove mathematically that the iterative algorithm has the total energy decaying property. The proposed algorithm is simple and easy to implement. Extensive numerical experiments in both two and three dimensions show that the proposed iteration algorithm converges in much fewer iterations and is more efficient than many existing methods. In addition, the numerical results show that the algorithm is very robust and insensitive to the initial guess and the parameters in the model.
\end{abstract}

AMS subject classifications: 35K93, 35K05, 65M12, 35Q35, 49Q10, 65M60, $76 \mathrm{~S} 05$

Key words: Topology optimization, Stokes flow, threshold dynamics method, mixed finite-element method.

\footnotetext{
*Corresponding author. Email addresses: chx@xmu.edu.cn (H. Chen), htleng@m.scnu.edu.cn (H. Leng), wangdong@cuhk.edu.cn (D. Wang), mawang@ust.hk (X.-P. Wang)
} 


\section{Introduction}

Topology optimization has become a significant problem due to its application in many industrial problems such as the optimization of transport vehicles and biomechanical structure. The process of topology optimization allows the introduction of new boundaries as part of the solution and is thus more flexible than shape optimization, which requires that the topology be predetermined. It is since the original work by Bendsøe and Kikuchi [8] on homogenization approach to topology optimization that many methods have been developed. For instance, region-based approaches: density interpolation [7], level-set method approach [5], topological derivatives [27], and phase field method [17]; contour-based approaches: evolutionary structural optimization (ESO) approaches [48] and the bi-directional schemes (BESO) [52]; and several others. Topology optimization was first applied to fluid mechanics by Borrvall and Petersson [9] by adapting the concept of density methods to Stokes flows. In [9], the total domain with fluid-solid regions was treated as a porous medium, the Brinkman flow was introduced to obtain a well-posed problem to minimize the total dissipation power, and the discrete optimization problem was further solved with the method of moving asymptotes (MMA) [37] to obtain the optimal designed regions for fluids and solids. Topology optimization in fluid mechanics has since been extended to the Darcy-Stokes flow model [47], Navier-Stokes flow [39], and non-Newtonian flow [33], and it has also been applied in the design of more complicated fluidic devices $[6,28,29]$.

The level set based approach [30] has been very popular in topology optimization. It was applied to fluidic topology optimization where the fluid-solid interface is described by the zero-level contour of a level set function. In [2], the authors proposed a method that combines the sensitivity analysis and the level set method for many problems in topology optimization. It has been subsequently extended to multi-phase structure optimization [3], optimal design [4], multiple loads structural optimization [5,46], and also fluid problems [22,39].

To ensure well-posedness and mesh-independent solutions, regularization is usually needed in topology optimization. Many robust regularization approaches were introduced. For example, regularizing the optimization problem by introducing fictitious interface energy [51] or penalizing the original objective function by perimeter control functions based on phase field method [34]. The level set based method can also be applied to solve topology optimization problems (e.g. [2] and the reference therein).

The existing numerical methods for solving the topology optimization for Stokes flow problem are quite mature. However, the efficiency of these methods can still be improved which is what we consider in this paper. When the MMA is applied to the topology optimization for Stokes flow [47], an additional sub-optimization problem needs to be solved and the parameters involved also need to be updated in each iteration. In the level set based methods for topology optimization for fluids [2,22], one needs to do either reinitialization/redistancing or some related techniques. These motivate us to design a new iterative algorithm based on the threshold dynamics approach from [13]. The new algorithm 
has two key merits compared with the existing methods. First, the algorithm decreases energy (objective functional) in each iteration step which implies unconditional stability of the iteration algorithm and faster convergence. Second, the fluid-solid regions are updated by convolution and a simple thresholding, thus the computational complexity (in addition to the usual Stokes solver) is optimal. From the numerical experiments presented in this paper, we see that the new algorithm has the faster convergence compared with the MMA and level set methods.

The threshold dynamics method developed by Merriman, Bence, and Osher (MBO) [26] is an efficient method for simulating the motion of an interface driven by its mean curvature. The method alternatively diffuses characteristic functions of regions and sharpens the result through thresholding to generate the interface motion implicitly. To be precise, let $D$ be domain of one phase with boundary $\Gamma=\partial D$ that separates phases. The domain $D$ and boundary $\Gamma$ are updated via two steps:

Step 1: Solve free space heat equation with initially constant temperature in domain $D$ and zero elsewhere in a short time $\tau$ by convolution with heat kernel,

$$
\phi(x)=G_{\tau} * \chi_{D},
$$

where $\chi_{D}$ is the characteristic function of $D$. Here,

$$
G_{\tau}=\frac{1}{(4 \pi \tau)^{d / 2}} \exp \left(-\frac{|x|^{2}}{4 \tau}\right)
$$

is the heat kernel where $d$ is spacial dimension of $D$.

Step 2: Obtain new domain $D^{*}$ and interface $\Gamma^{*}=\partial D^{*}$ by thresholding,

$$
D^{*}=\left\{x: \phi(x) \geq \frac{1}{2}\right\} .
$$

The convergence of this algorithm to mean curvature flow was well established $[16,38]$. Esedoglu and Otto [13] gave a variational formulation for the method and generalized it to multiphase problems with arbitrary surface tensions. They observed that

$$
J_{\tau}(D)=\sqrt{\frac{\pi}{\tau}} \int \chi_{D^{c}} G_{\tau} * \chi_{D} d x
$$

is a non-local approximation to perimeter of boundary of $D$ and showed that threshold dynamics scheme dissipates this functional $J_{\tau}$ at each iteration. Moreover, they gave the following minimizing movement interpretation:

$$
D^{*}=\underset{\bar{D}}{\arg \min _{\bar{\tau}}} J_{\tau}(\bar{D})+g\left(\chi_{\bar{D}}-\chi_{D}\right),
$$

where

$$
g(a)=\sqrt{\frac{\pi}{\tau}} \int a G_{\tau} * a d x=\sqrt{\frac{\pi}{\tau}} \int\left[G_{\tau / 2} * a\right]^{2} d x
$$


is a positive definite quadratic functional measuring the distance between $\bar{D}$ and $D$. Essentially, the threshold dynamics method is reformulated into an iterative method for domain optimization problem with the non-local interface energy $J_{\tau}(D)$ as the objective function and the admissible set being the characteristic functions of domain $D$. The method has attracted considerable attention due to its simplicity and unconditional stability. It has since been extended to deal with many other applications, including the problem of area-preserving or volume-preserving interface motion [36], image processing [15, 25, 42, 44], problems of anisotropic interface motion [12], surface reconstruction [40], the wetting problem on solid surfaces [24, 41, 45, 49,50], and auction dynamics [21]. Various algorithms and rigorous error analysis have been introduced to refine and extend the original MBO method and related methods for these problems (see, e.g., [14, 20,35]). Adaptive methods have also been used to accelerate this type of method [23] based on nonuniform fast Fourier transform. A generalized manifold-valued threshold dynamics method was developed by $[31,32,43]$.

In this paper, we generalize the threshold dynamics approach to the topology optimization for fluids. In our approach, we minimize the total energy (objective function) consisting of the dissipation power in the fluid and the perimeter regularization, subject to a fluid volume constraint and the Stokes equation for the velocity field. The perimeter term is based on convolution of the heat kernel with the characteristic functions of the fluid regions. The porous medium approach is used in our algorithm, and we introduce the Brinkman equation, which "interpolates" between the Stokes equation in the flow region and some Darcy flow through a porous medium (a weakened nonfluid region). We then solve the Brinkman equation for the whole domain by the standard mixed finiteelement method and update the fluid-solid regions by convolution and with a simple thresholding step. In particular, the convolutions can be efficiently computed on a uniform grid by fast Fourier transform (FFT) with the an optimal complexity of $\mathcal{O}(N \log N)$.

An efficient iterative thresholding scheme is derived to minimize an approximate total energy (objective function) with constraints. The main observation is that our objective function is concave with respect to the characteristic function of the domain when the state variable (velocity field) is fixed. This allows us to design an iterative method to minimize the objective function in a simple and robust way and accelerates the minimization of the energy in the method. The proposed method is easy to implement and numerical results show that the method is efficient, robust and insensitive to the initial guess and parameters. In particular, we further prove the unconditional energy-decaying property of the proposed algorithm which is usually not theoretically guaranteed in level set based methods. Numerical results show that the number of iterations needed to reach the stationary state is greatly reduced when compared to some level set based method or the MMA. Our method can be easily extended to cases with arbitrary numbers of phases in multi-phase topology optimization. Overall, the new method is simple, easy to implement, unconditionally stable, and efficient. This paper is the first application of the threshold dynamics to the topology optimization for fluids in a simple case. We expect that the new method can be generalized to more complicated multi-physics related topol- 
ogy optimization problems.

The paper is organized as follows. In Section 2, we show the mathematical model. In Section 3, we introduce an approximate energy to the total energy and derive an efficient iterative thresholding method. The unconditional stability of the method (i.e., the energy decaying property) is proved in Section 4. We discuss the numerical implementation in Section 5 and verify the efficiency and the energy decaying property of the algorithm in Section 6. We make conclusions, and discuss some ideas for future work in Section 7.

\section{Mathematical model}

In this section, we consider the mathematical model for topology optimization for fluids in Stokes flow. Denote $\Omega \in \mathbb{R}^{d}(d=2,3)$ the computational domain, which is fixed throughout optimization, and assume that $\Omega$ is a bounded Lipschitz domain with an outer unit normal $\mathbf{n}$ such that $\mathbb{R}^{d} \backslash \bar{\Omega}$ is connected. Furthermore, we denote $\Omega_{0} \subset \Omega$ the domain of the fluid, which is a Caccioppoli set whose boundary is measurable and has a (at least locally) finite measure (cf. [19]), and $\Omega \backslash \Omega_{0} \in \Omega$ the solid domain. Throughout the paper, we use the standard notations and definitions for Sobolev spaces (cf. [1]). Our goal is to determine an optimal shape of $\Omega_{0}$ that minimizes the following objective functional consisting of the total potential power and a perimeter regularization term,

$$
\min _{\left(\Omega_{0}, \mathbf{u}\right)} J_{0}\left(\Omega_{0}, \mathbf{u}\right)=\int_{\Omega}\left(\frac{\mu}{2}|D \mathbf{u}|^{2}-\mathbf{u} \cdot \mathbf{f}\right) d \mathbf{x}+\gamma|\Gamma|
$$

subject to

$$
\begin{aligned}
\nabla \cdot \mathbf{u}=0, & \text { in } \Omega_{0}, \\
\nabla p-\nabla \cdot(\mu \nabla \mathbf{u})=\mathbf{f}, & \text { in } \Omega_{0}, \\
\mathbf{u}=0, & \text { in } \Omega \backslash \bar{\Omega}_{0} \text { and on } \partial \Omega_{0}, \\
\left.\mathbf{u}\right|_{\partial \Omega}=\mathbf{u}_{D}, & \text { on } \partial \Omega, \\
\left|\Omega_{0}\right|=\beta|\Omega|, & \text { with a fixed parameter } \beta \in(0,1) .
\end{aligned}
$$

Here, $\mathbf{u}: \Omega \rightarrow \mathbb{R}^{d}, D \mathbf{u}$ is the distributional derivative of $\mathbf{u}, \mu$ is the dynamic viscosity of the fluid, $p$ is the pressure, $\mathbf{u}_{D}: \partial \Omega \rightarrow \mathbb{R}^{d}$ is a given function, $\mathbf{f}: \Omega \rightarrow \mathbb{R}^{d}$ is a given external force, $|\Gamma|$ is the perimeter of the boundary of $\Gamma=\partial \Omega_{0}$, and $\gamma>0$ is a weighting parameter.

\section{Derivation of the algorithm}

In this section, we develop an efficient threshold dynamics method for the topology optimization problem discussed in (2.1) and (2.2) for fluids in Stokes flow. Note that the goal is to determine the optimal interface between liquid and solid that minimizes functional (2.1) subject to constraints (2.2). Motivated by the idea from the threshold dynamics methods developed by $[13,42,45,49]$, we use the indicator functions for the fluid region and the solid region to implicitly represent the interface. 


\subsection{Approximate energy}

Define an admissible set $\mathcal{B}$ as follows:

$$
\mathcal{B}:=\left\{\left(v_{1}, v_{2}\right) \in B V(\Omega) \mid v_{i}(x)=\{0,1\}, v_{1}(x)+v_{2}(x)=1 \text { a.e. in } \Omega, \text { and } \int_{\Omega} v_{1} d \mathbf{x}=V_{0}\right\},
$$

where $B V(\Omega)$ is the vector space of functions with bounded variation in $\Omega$, and $V_{0}$ is the fixed volume of the fluid region. We introduce $\chi_{1}(\mathbf{x})$ to denote the indicator function of the fluid region $\Omega_{0}$, i.e.,

$$
\chi_{1}(\mathbf{x}):= \begin{cases}1, & \text { if } \mathbf{x} \in \Omega_{0}, \\ 0, & \text { otherwise, }\end{cases}
$$

and $\chi_{2}(\mathbf{x})$ as the indicator function of $\Omega \backslash \Omega_{0}$, i.e., $\chi_{2}(\mathbf{x})=1-\chi_{1}(\mathbf{x})$. The interface $\Gamma$ is then implicitly represented by $\chi_{1}$ and $\chi_{2}$. Let $\chi=\left(\chi_{1}, \chi_{2}\right)$ and we have $\chi \in \mathcal{B}$. The perimeter of the interface $\Gamma$ is then approximated by,

$$
|\Gamma| \approx \sqrt{\frac{\pi}{\tau}} \int_{\Omega} \chi_{1} G_{\tau} * \chi_{2} d \mathbf{x}
$$

where $G_{\tau}(\mathbf{x})=\frac{1}{(4 \pi \tau)^{\frac{d}{2}}} \exp \left(-\frac{|\mathbf{x}|^{2}}{4 \tau}\right)$ is the Gaussian kernel (see [13] for more details on this approximation).

We solve the optimization problem (2.1)-(2.2) iteratively. At each iteration, one must solve the Stokes equation in the fluid domain, which is changing in the iteration. It is more convenient numerically to use the porous medium approach as in $[11,17]$. The idea is to "interpolate" between the Stokes equation in the fluid domain (i.e., $\left\{\mathbf{x} \mid \chi_{1}(\mathbf{x})=1\right\}$ ) and $\mathbf{u}=0$ in the solid domain (i.e., $\left\{\mathbf{x} \mid \chi_{2}(\mathbf{x})=1\right\}$ ) by introducing an additional penalization term,

$$
\begin{aligned}
\nabla \cdot \mathbf{u}=0, & \text { in } \Omega, \\
\nabla p-\nabla \cdot(\mu \nabla \mathbf{u})+\alpha(\mathbf{x}) \mathbf{u}=\mathbf{f}, & \text { in } \Omega, \\
\left.\mathbf{u}\right|_{\partial \Omega}=\mathbf{u}_{D}, & \text { on } \partial \Omega .
\end{aligned}
$$

Here, $\alpha(\mathbf{x})$ is a smooth function that varies between 0 and $\bar{\alpha}_{\tau}$ through a thin interface layer around $\Gamma$, and $\bar{\alpha}_{\tau}^{-1}$ is the permeability. In the current representation of the interface, we use the 0.5 level set of $\phi=G_{\tau} * \chi_{2}$ to approximate the position of the interface $\Gamma$. It is well known that such $\phi$ is a smooth function between $[0,1]$ and admits a change from 0 to 1 in an $\mathcal{O}(\sqrt{\tau})$ thin layer. Hence, $\alpha$ is given by

$$
\alpha=\bar{\alpha}_{\tau} \phi=\bar{\alpha}_{\tau} G_{\tau} * \chi_{2} .
$$

In the limiting model (i.e., $\tau \searrow 0$ ), $\bar{\alpha}_{\tau}$ should be set as $+\infty$ to make the constraints $\{\mathbf{u}=$ 0 in $\Omega \backslash \Omega_{0}$ \} satisfied. Also, to ensure that the velocity vanishes outside the fluid domain 
when $\tau \searrow 0$, we add a penalty term

$$
\int_{\Omega} \frac{\alpha}{2}|\mathbf{u}|^{2} d x=\int_{\Omega} \frac{\bar{\alpha}_{\tau} G_{\tau} * \chi_{2}}{2}|\mathbf{u}|^{2} d x
$$

to the objective functional. In the follows, for numerical consideration, we fix $\bar{\alpha}_{\tau}$ as a sufficiently large constant, $\bar{\alpha}$. In this porous media approach, the system (3.3) is solved for a fixed domain $\Omega$.

Finally, combining (2.1), (3.2), (3.4), and the penalty term, we arrive at the following approximate objective functional

$$
J^{\tau}(\chi, \mathbf{u})=\int_{\Omega}\left(\frac{\mu}{2}|D \mathbf{u}|^{2}+\frac{\bar{\alpha}}{2}|\mathbf{u}|^{2} G_{\tau} * \chi_{2}-\mathbf{u} \cdot \mathbf{f}+\gamma \sqrt{\frac{\pi}{\tau}} \chi_{1} G_{\tau} * \chi_{2}\right) d \mathbf{x} .
$$

Remark 3.1. For simplicity, we use the same $\tau$ in the second and the fourth terms of the above approximate energy. Indeed, one can also use different values of $\tau$ in the two terms and the property of the algorithm will be similar.

Now, we consider the following approximate formulation of the problem

$$
\min _{(\chi, \mathbf{u})} J^{\tau}(\chi, \mathbf{u}) \text {, subject to } \chi=\left(\chi_{1}, \chi_{2}\right) \in \mathcal{B} \text { and } \mathbf{u} \text { satisfies (3.3). }
$$

In the following, we give the derivation of the threshold dynamics scheme to solve (3.6).

\subsection{Derivation of the scheme}

In this section, based on [13], we use a coordinate descent algorithm to minimize the approximate energy (3.5) with constraints (3.3). A similar idea has been applied in the design of a threshold dynamics method for image segmentation [42]. Given an initial guess $\chi^{0}=\left(\chi_{1}^{0}, \chi_{2}^{0}\right)$, we compute a series of minimizers

$$
\mathbf{u}^{0}, \chi^{1}, \mathbf{u}^{1}, \chi^{2}, \cdots, \mathbf{u}^{k}, \chi^{k+1}, \cdots
$$

such that

$$
\begin{aligned}
& \left.\mathbf{u}^{k}=\underset{\mathbf{u} \in \mathcal{S}}{\arg \min ^{\tau}} J^{k}, \mathbf{u}\right), \\
& \chi^{k+1}=\underset{\chi \in \mathcal{B}}{\arg \min ^{\tau}\left(\chi, \mathbf{u}^{k}\right),},
\end{aligned}
$$

for $k=0,1,2, \cdots$. Here, the admissible set $\mathcal{S}$ is defined as

$$
\mathcal{S}:=\left\{\mathbf{u} \in H_{\mathbf{u}_{D}}^{1}\left(\Omega, \mathbb{R}^{d}\right) \mid \nabla \cdot \mathbf{u}=0\right\},
$$

where $H_{\mathbf{u}_{D}}^{1}\left(\Omega, \mathbb{R}^{d}\right)=\left\{\mathbf{u} \in H^{1}\left(\Omega, \mathbb{R}^{d}\right)|\mathbf{u}|_{\partial \Omega}=\mathbf{u}_{D}\right\}$, and $\mathcal{B}$ is defined in (3.1). 
Given the $k$-th iteration $\chi^{k}$, we first solve (3.7) to get the $\mathbf{u}^{k}$. It is easy to see that the constraint minimization problem is equivalent to the following

$$
\mathbf{u}^{k}=\arg \min _{\mathbf{u} \in H_{\mathbf{u}_{D}}^{1}\left(\Omega, \mathbb{R}^{d}\right)} J^{\tau}\left(\chi^{k}, \mathbf{u}\right)+\int_{\Omega} p \nabla \cdot \mathbf{u} d \mathbf{x},
$$

with $p$ as a Lagrangian multiplier. Variation of the above functional leads to the following Brinkman equation. That is, $\mathbf{u}^{k}$ can be obtained by solving

$$
\begin{cases}\nabla \cdot \mathbf{u}=0, & \text { in } \Omega, \\ \nabla p-\nabla \cdot(\mu \nabla \mathbf{u})+\alpha^{k} \mathbf{u}=\mathbf{f}, & \text { in } \Omega, \\ \left.\mathbf{u}\right|_{\partial \Omega}=\mathbf{u}_{D}, & \end{cases}
$$

where $\alpha^{k}=\frac{\bar{\alpha}}{2} G_{\tau} * \chi_{2}^{k}$. Because $J^{\tau}\left(\chi^{k}, \mathbf{u}\right)$ is convex in $\mathbf{u}$, the solution $\left(\mathbf{u}^{k}, p^{k}\right)$ of (3.9) is a minimizer of $J^{\tau}\left(\chi^{k}, \mathbf{u}\right)$. The following lemma shows the existence of $\mathbf{u}$ for the system (3.9) for a given $\chi \in \mathcal{B}$.

Lemma 3.1 ([17, 18]). For every $\chi \in \mathcal{B}$, some $\mathbf{u} \in H_{\mathbf{u}_{D}}^{1}\left(\Omega, \mathbb{R}^{d}\right)$ exist that satisfy $\nabla \cdot \mathbf{u}=0$ such that

$$
\int_{\Omega} \mu \nabla \mathbf{u} \cdot \nabla \mathbf{v}+\alpha \mathbf{u} \cdot \mathbf{v} d \mathbf{x}=\int_{\Omega} \mathbf{f} \cdot \mathbf{v} d \mathbf{x}, \text { forall } \mathbf{v} \in V
$$

where $\boldsymbol{V}:=\left\{\boldsymbol{v} \in H_{0}^{1}\left(\Omega, \mathbb{R}^{d}\right) \mid \nabla \cdot \boldsymbol{v}=0\right\}$.

Given $\mathbf{u}^{k}$, we now rewrite the objective functional $J^{\tau}(\chi, \mathbf{u})$ into $\tilde{J}^{\tau, k}(\chi)$ as follows:

$$
\begin{aligned}
\tilde{J}^{\tau, k}(\chi) & :=J^{\tau}\left(\chi, \mathbf{u}^{k}\right) \\
& =\int_{\Omega} \frac{\bar{\alpha}}{2} \chi_{2} G_{\tau} *\left|\mathbf{u}^{k}\right|^{2} d \mathbf{x}+\gamma \sqrt{\frac{\pi}{\tau}} \int_{\Omega} \chi_{1} G_{\tau} * \chi_{2} d \mathbf{x}+\int_{\Omega} \frac{\mu}{2}\left|D \mathbf{u}^{k}\right|^{2}-\mathbf{u}^{k} \cdot \mathbf{f} d \mathbf{x} .
\end{aligned}
$$

The next step is to find $\chi^{k+1}$ such that

$$
\chi^{k+1}=\underset{\chi \in \mathcal{B}}{\arg \min } \tilde{J}^{\tau, k}(\chi) .
$$

It is the minimization of a concave functional on a nonconvex admissible set $\mathcal{B}$. However, we can relax it to a problem defined on a convex admissible set by finding $r^{k+1}$ such that

$$
\boldsymbol{r}^{k+1}=\underset{r \in \mathcal{H}}{\arg \min } \tilde{J}^{\tau, k}(\boldsymbol{r}),
$$

where $\mathcal{H}$ is the convex hull of $\mathcal{B}$ defined as follows:

$\mathcal{H}:=\left\{\left(v_{1}, v_{2}\right) \in B V(\Omega) \mid v_{i}(x) \in[0,1], i=1,2\right.$, and $v_{1}(x)+v_{2}(x)=1$ a.e. in $\left.\Omega, \int_{\Omega} v_{1} d \mathbf{x}=V_{0}\right\}$.

The following lemma shows that the relaxed problem (3.13) is equivalent to the original problem (3.12). Therefore, we can solve the relaxed problem (3.13) instead. 
Lemma 3.2. Let $\mathbf{u} \in H_{\mathbf{u}_{D}}^{1}\left(\Omega, \mathbb{R}^{d}\right)$ be a given function and $\boldsymbol{r}=\left(r_{1}, r_{2}\right)$. Then

$$
\underset{r \in \mathcal{H}}{\operatorname{argmin}} \tilde{J}^{\tau, k}(\boldsymbol{r})=\underset{r \in \mathcal{B}}{\arg \min } \tilde{J}^{\tau, k}(\boldsymbol{r}) .
$$

Proof. Let $\tilde{r}=\left(\tilde{r}_{1}, \tilde{r}_{2}\right) \in \mathcal{H}$ be a minimizer of the functional $\tilde{J}^{\tau, k}(\boldsymbol{r})$ on $\mathcal{H}$. Because $\mathcal{B} \subset \mathcal{H}$, we have

$$
\tilde{J}^{\tau, k}(\tilde{\boldsymbol{r}})=\min _{\boldsymbol{r} \in \mathcal{H}} \tilde{J}^{\tau, k}(\boldsymbol{r}) \leq \min _{\boldsymbol{r} \in \mathcal{B}} \tilde{J}^{\tau, k}(\boldsymbol{r}) .
$$

Therefore, we need only prove that $\tilde{r} \in \mathcal{B}$.

We proceed by contradiction. If $\tilde{\boldsymbol{r}} \notin \mathcal{B}$, there is a set $A \in \Omega$ and a constant $0<C_{0}<\frac{1}{2}$, such that $|A|>0$ and

$$
0<C_{0}<\tilde{r}_{1}(\mathbf{x}), \tilde{r}_{2}(\mathbf{x})<1-C_{0}, \text { for all } \mathbf{x} \in A \text {. }
$$

We divide $A$ into two sets $A=A_{1} \cup A_{2}$ such that $A_{1} \cap A_{2}=\varnothing$ and $\left|A_{1}\right|=\left|A_{2}\right|=|A| / 2$. Denote $\boldsymbol{r}^{t}=\left(r_{1}^{t}, r_{2}^{t}\right)$ where $r_{1}^{t}=\tilde{r}_{1}+t \chi_{A_{1}}-t \chi_{A_{2}}$ and $r_{2}^{t}=\tilde{r}_{2}-t \chi_{A_{1}}+t \chi_{A_{2}}$ with $\chi_{A_{1}}$ and $\chi_{A_{2}}$ being the indicator functions of the domain $A_{1}$ and $A_{2}$, respectively. When $0<t<C_{0}$, we have $0<r_{1}^{t}, r_{2}^{t}<1$ and

$$
r_{1}^{t}+r_{2}^{t}=\tilde{r}_{1}+\tilde{r}_{2}=1, \quad \text { and } \quad \int_{\Omega} r_{1}^{t} d \mathbf{x}=\int_{\Omega} \tilde{r}_{1} d \mathbf{x}=V_{0} .
$$

This implies that $r^{t} \in \mathcal{H}$. Furthermore, direct computations give,

$$
\begin{aligned}
\frac{d^{2}}{d t^{2}} \tilde{J}^{\tau, k}(\boldsymbol{r}) & =2 \gamma \frac{\sqrt{\pi}}{\sqrt{\tau}} \int_{\Omega} \frac{d}{d t} r_{1}^{t} G_{\tau} * \frac{d}{d t} r_{2}^{t} d \mathbf{x} \\
& =2 \gamma \frac{\sqrt{\pi}}{\sqrt{\tau}} \int_{\Omega}\left(\chi_{A_{1}}-\chi_{A_{2}}\right) G_{\tau} *\left(\chi_{A_{2}}-\chi_{A_{1}}\right) d \mathbf{x} \\
& =-2 \gamma \frac{\sqrt{\pi}}{\sqrt{\tau}} \int_{\Omega}\left(\chi_{A_{1}}-\chi_{A_{2}}\right) G_{\tau} *\left(\chi_{A_{1}}-\chi_{A_{2}}\right) d \mathbf{x} \\
& =-2 \gamma \frac{\sqrt{\pi}}{\sqrt{\tau}} \int_{\Omega}\left(G_{\tau / 2} *\left(\chi_{A_{1}}-\chi_{A_{2}}\right)\right)\left(G_{\tau / 2} *\left(\chi_{A_{1}}-\chi_{A_{2}}\right)\right) d \mathbf{x} \\
& \leq 0 .
\end{aligned}
$$

The penultimate step comes from the fact that the heat kernel is a self-adjoint operator and forms a semigroup with various values of $\tau$. From the above inequality, the functional is concave on the point $\tilde{r}$. Thus, $\tilde{r}$ cannot be a minimizer of the functional. This contradicts the assumption.

Now, we show that (3.13) can be solved with a simple threshold dynamics method. Because $\tilde{J}^{\tau, k}(\boldsymbol{r})$ is quadratic in $\boldsymbol{r}$ and concave, we first linearize the energy $\tilde{J}^{\tau, k}(\boldsymbol{r})$ at $\boldsymbol{r}^{k}$ by

$$
\tilde{J}^{\tau, k}(\boldsymbol{r}) \approx \tilde{J}^{\tau, k}\left(\boldsymbol{r}^{k}\right)+\mathcal{L}_{\boldsymbol{r}^{k}}^{\tau, k}\left(\boldsymbol{r}-\boldsymbol{r}^{k}\right),
$$


where

$$
\begin{aligned}
\mathcal{L}_{r^{k}}^{\tau, k}(\boldsymbol{r}) & =\int_{\Omega}\left(\gamma \sqrt{\frac{\pi}{\tau}} r_{1} G_{\tau} * r_{2}^{k}+\gamma \sqrt{\frac{\pi}{\tau}} r_{2} G_{\tau} * r_{1}^{k}+r_{2} \frac{\bar{\alpha}}{2} G_{\tau} *\left|\mathbf{u}^{k}\right|^{2}\right) d \mathbf{x} \\
& =\int_{\Omega}\left(r_{1} \phi_{1}+r_{2} \phi_{2}\right) d \mathbf{x} .
\end{aligned}
$$

Here $\phi_{1}=\gamma \sqrt{\frac{\pi}{\tau}} G_{\tau} * r_{2}^{k}$ and $\phi_{2}=\frac{\bar{\alpha}}{2} G_{\tau} *\left|\mathbf{u}^{k}\right|^{2}+\gamma \sqrt{\frac{\pi}{\tau}} G_{\tau} * r_{1}^{k}$. Then (3.13) can be approximately reformulated into

$$
\chi^{k+1}=\arg \min _{\boldsymbol{r} \in \mathcal{H}} \mathcal{L}_{r^{k}}^{\tau, k}(\boldsymbol{r})=\arg \min _{\boldsymbol{r} \in \mathcal{H}} \int_{\Omega}\left(r_{1} \phi_{1}+r_{2} \phi_{2}\right) d \mathbf{x} .
$$

The following lemma, in particular, (3.21) shows that (3.18) can be solved in a pointwise manner by

$$
\begin{cases}\chi_{1}^{k+1}(\mathbf{x})=1 \text { and } \chi_{2}^{k+1}(\mathbf{x})=0, & \text { if } \phi_{1}(\mathbf{x})<\phi_{2}(\mathbf{x})+\delta, \\ \chi_{1}^{k+1}(\mathbf{x})=0 \text { and } \chi_{2}^{k+1}(\mathbf{x})=1, & \text { otherwise, }\end{cases}
$$

where $\delta$ is chosen as a constant such that $\int_{\Omega} \chi_{1}^{k+1} d \mathbf{x}=V_{0}$.

Lemma 3.3. Let $\phi_{1}=\gamma \sqrt{\frac{\pi}{\tau}} G_{\tau} * \chi_{2}^{k}, \phi_{2}=\frac{\bar{\alpha}}{2} G_{\tau} *\left|\mathbf{u}^{k}\right|^{2}+\gamma \sqrt{\frac{\pi}{\tau}} G_{\tau} * \chi_{1}^{k}$,

$$
D_{1}^{k+1}=\left\{\mathbf{x} \in \Omega \mid \phi_{1}-\phi_{2}<\delta\right\}
$$

for some $\delta$ such that $\left|D_{1}^{k+1}\right|=V_{0}$, and $D_{2}^{k+1}=\Omega \backslash D_{1}^{k+1}$. Then for $\chi^{k+1}=\left(\chi_{1}^{k+1}, \chi_{2}^{k+1}\right)$ with $\chi_{1}^{k+1}=\chi_{D_{1}^{k+1}}$ and $\chi_{2}^{k+1}=1-\chi_{1}^{k+1}$, we have

$$
\mathcal{L}_{\chi^{k}}^{\tau, k}\left(\chi^{k+1}\right) \leq \mathcal{L}_{\chi^{k}}^{\tau, k}\left(\chi^{k}\right)
$$

for all $\tau>0$.

Proof. Because $\mathcal{L}_{\chi^{k}}^{\tau, k}$ is a linear functional, we only need to prove that there holds

$$
\mathcal{L}_{\chi^{k}}^{\tau, k}\left(\chi^{k+1}\right) \leq \mathcal{L}_{\chi^{k}}^{\tau, k}(\chi)
$$

for all $\chi=\left(\chi_{1}, \chi_{2}\right) \in \mathcal{B}$.

For each $\left(\chi_{1}, \chi_{2}\right) \in \mathcal{B}$, we know $\chi_{1}=\chi_{\hat{D}_{1}}$ and $\chi_{2}=\chi_{\hat{D}_{2}}$ for some open sets $\hat{D}_{1}, \hat{D}_{2}$ in $\Omega$, such that $\hat{D}_{1} \cap \hat{D}_{2}=\varnothing, \hat{D}_{1} \cup \hat{D}_{2}=\Omega$ and $\left|\hat{D}_{1}\right|=V_{0}$. Let $A_{1}=\hat{D}_{1} \backslash D_{1}^{k+1}=D_{2}^{k+1} \backslash \hat{D}_{2}$ and $A_{2}=\hat{D}_{2} \backslash D_{2}^{k+1}=D_{1}^{k+1} \backslash \hat{D}_{1}$. We must have $\left|A_{1}\right|=\left|A_{2}\right|$ due to the volume conservation property. Because $A_{1} \subset D_{2}^{k+1}$, we have

$$
\phi_{1}(\mathbf{x})-\phi_{2}(\mathbf{x}) \geq \delta, \quad \chi_{1}^{k+1}(\mathbf{x})-\chi_{1}(\mathbf{x})=-1, \quad \forall \mathbf{x} \in A_{1} .
$$


Similarly, because $A_{2} \subset D_{1}^{k+1}$, we have

$$
\phi_{1}(\mathbf{x})-\phi_{2}(\mathbf{x})<\delta, \quad \chi_{1}^{k+1}(\mathbf{x})-\chi_{1}(\mathbf{x})=1, \quad \forall \mathbf{x} \in A_{2} .
$$

Therefore, using $\chi_{1}^{k+1}-\chi_{1}+\chi_{2}^{k+1}-\chi_{2}=0$, we have

$$
\begin{aligned}
\mathcal{L}_{\chi^{k}}^{\tau, k}\left(\chi^{k+1}\right)-\mathcal{L}_{\chi^{k}}^{\tau, k}(\chi) & =\gamma \sqrt{\frac{\pi}{\tau}} \int_{\Omega}\left(\chi_{1}^{k+1}-\chi_{1}\right) \phi_{1}+\left(\chi_{2}^{k+1}-\chi_{2}\right) \phi_{2} d \mathbf{x} \\
& =\gamma \sqrt{\frac{\pi}{\tau}} \int_{\Omega}\left(\chi_{1}^{k+1}-\chi_{1}\right)\left(\phi_{1}-\phi_{2}\right) d \mathbf{x} \\
& =\gamma \sqrt{\frac{\pi}{\tau}} \int_{\Omega}\left(\chi_{A_{2}}\left(\phi_{1}-\phi_{2}\right)-\chi_{A_{1}}\left(\phi_{1}-\phi_{2}\right)\right) d \mathbf{x} \\
& \leq \gamma \sqrt{\frac{\pi}{\tau}} \int_{\Omega}\left(\chi_{A_{2}} \delta-\chi_{A_{1}} \delta\right) d \mathbf{x}=\gamma \sqrt{\frac{\pi}{\tau}} \delta\left(\left|A_{2}\right|-\left|A_{1}\right|\right)=0 .
\end{aligned}
$$

This completes the proof.

To determine the value of $\delta$, one can treat $\int_{\Omega} \chi_{1}^{k+1} d \mathbf{x}-V_{0}$ as a function of $\delta$ and use an iteration method (e.g., bisection method or Newton's method) to find the root of $\int_{\Omega} \chi_{1}^{k+1} d \mathbf{x}-V_{0}=0$. When $\Omega$ is equipped with a uniform discretization, an efficient method is the quick-sort technique proposed in [49]. Assume we have a uniform discretization of $\Omega$ with grid size $h$, we can approximate $\int_{\Omega} \chi_{1}^{k+1} d \mathbf{x}$ by $M h^{2}$, we then sort the values of $\phi_{1}-\phi_{2}$ in an ascending order and simply set $\chi_{1}^{k+1}=1$ on the first $M$ points and $\chi_{2}^{k+1}=1$ on the other points.

Remark 3.2. In many implementations, one may solve Stokes equation on nonuniform grid points. To preserve the volume for the discretization on nonuniform grids, although the volume cannot be simply approximated by the number of grid points times the size of each cell, a similar technique can be applied. One can still sort the values of $\phi_{1}-\phi_{2}$ in ascending order, save the index into $\mathcal{S}$, calculate the integrating weight at each grid point into $\mathcal{V}$, and set $V=0$ and $i=0$. Then, $\delta$ can be simply found by:

$$
\text { while } V<V_{0} ; i \leftarrow i+1 ; V=V+\mathcal{V}(\mathcal{S}(i)) ; \text { end } ; \delta=\phi_{1}(\mathcal{S}(i+1))-\phi_{2}(\mathcal{S}(i+1)) \text {. }
$$

Now, we are led to a threshold dynamics algorithm for topology optimization problem (3.6) for fluids in Stokes flow in Algorithm 1.

Remark 3.3. We note that in the original MBO method, on one hand, the algorithm can be easily stuck when $\tau$ is very small because, in the discretized space, $\tau$ is so small that no point can switch from one phase to another (i.e., $\chi_{1}$ changes from 0 to 1 or 1 to 0 ) at one iteration step. On the other hand, with a large $\tau$, the interface can easily move but creates large error. Hence, we apply the adaptive in time technique [49] in numerical experiments by modifying Algorithm 1 into an adaptive algorithm by adjusting $\tau$ during 


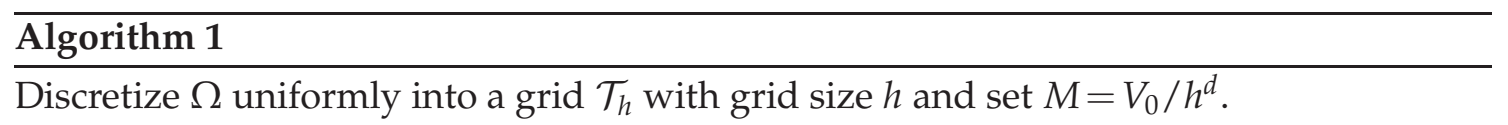

Step 1. Input: Set $\tau>0, \bar{\alpha}>0, k=0$, a tolerance parameter $t o l>0$ and give the initial guess $\chi^{0} \in \mathcal{B}$.

Step 2. Iterative solution: Given $\chi^{k}$,

1. Update u. Solve the Brinkman flow equations

$$
\begin{cases}\nabla \cdot \mathbf{u}=0, & \text { in } \Omega \\ \nabla p-\nabla \cdot(\mu \nabla \mathbf{u})+\alpha\left(\chi^{k}\right) \mathbf{u}=\mathbf{f}, & \text { in } \Omega, \\ \left.\mathbf{u}\right|_{\partial \Omega}=\mathbf{u}_{D}, & \end{cases}
$$

by mixed finite-element method to get $\mathbf{u}^{k}$, where $\alpha\left(\chi^{k}\right)=\bar{\alpha} G_{\tau} * \chi_{2}^{k}$.

2. Update $\chi$. Evaluate

$$
\left\{\begin{array}{l}
\phi_{1}=\gamma \sqrt{\frac{\pi}{\tau}} G_{\tau} * \chi_{2}^{k} \\
\phi_{2}=\frac{\bar{\alpha}}{2} G_{\tau} *\left|\mathbf{u}^{k}\right|^{2}+\gamma \sqrt{\frac{\pi}{\tau}} G_{\tau} * \chi_{1}^{k}
\end{array}\right.
$$

Sort the values of $\phi_{1}-\phi_{2}$ in an ascending order, and set $\chi_{1}^{k+1}=1$ on the first $M$ points and $\chi_{2}^{k+1}=1$ on the other points.

3. Compute $e_{\chi}^{k}=\left\|\chi_{1}^{k+1}-\chi_{1}^{k}\right\|_{2}$. If $e_{\chi}^{k}=0$, stop the iteration and go to the output step. Otherwise, let $k+1 \rightarrow k$ and go to Step 2 to continue the iteration.

Step 3. Output: A function $\chi \in \mathcal{B}$ that approximately solves (3.6).

the iterations. Specifically, if $e_{\chi}^{k}=0$, we set a new $\tau:=\eta \tau$ with $\eta \in(0,1)$ for the next iteration and iterate to the stationary solution with the fixed new $\tau$. When decreasing the value of $\tau$ makes no change on the solution, we then stop this process.

Remark 3.4. As for the efficiency, because such approximation of the regularization term using the concave functional, we find the minimizer of the linear approximation which is the optimal choice to minimize the linearized functional. Moreover, the minimizer can give a smaller value of the energy because the graph of a concave functional is always below its linear approximation. This accelerates the minimization of the energy in the method.

\section{Stability analysis}

In this section, we prove the unconditional stability property of the proposed algorithm. Specifically, for the series of minimizers

$$
\mathbf{u}^{0}, \chi^{1}, \mathbf{u}^{1}, \chi^{2}, \cdots, \mathbf{u}^{k}, \chi^{k+1}, \cdots,
$$


computed by Algorithm 1, we prove

$$
J^{\tau}\left(\chi^{k+1}, \mathbf{u}^{k+1}\right) \leq J^{\tau}\left(\chi^{k}, \mathbf{u}^{k}\right)
$$

for all $\tau>0$.

We first introduce Lemma 4.1 which leads us to $J^{\tau}\left(\chi^{k+1}, \mathbf{u}^{k}\right) \leq J^{\tau}\left(\chi^{k}, \mathbf{u}^{k}\right)$ for all $\tau>0$. Lemma 4.1. For a fixed $\mathbf{u}^{k}$, let $\chi^{k+1}$ be the $k+1$-th iteration derived from Algorithm 1 , we have

$$
J^{\tau}\left(\chi^{k+1}, \mathbf{u}^{k}\right) \leq J^{\tau}\left(\chi^{k}, \mathbf{u}^{k}\right)
$$

for all $\tau>0$.

Proof. From the linearization of $\tilde{J}^{\tau, k}\left(\chi^{k}\right)$ in (3.16), we have

$$
\begin{aligned}
& J^{\tau}\left(\chi^{k}, \mathbf{u}^{k}\right)=\mathcal{L}_{\chi^{k}}^{\tau, k}\left(\chi^{k}\right)-\gamma \sqrt{\frac{\pi}{\tau}} \int_{\Omega} \chi_{1}^{k} G_{\tau} * \chi_{2}^{k} d \mathbf{x}+\int_{\Omega} \frac{\mu}{2}\left|D \mathbf{u}^{k}\right|^{2}-\mathbf{u}^{k} \cdot \mathbf{f} d \mathbf{x}, \\
& J^{\tau}\left(\chi^{k+1}, \mathbf{u}^{k}\right)= \mathcal{L}_{\chi^{k}}^{\tau, k}\left(\chi^{k+1}\right)-\gamma \sqrt{\frac{\pi}{\tau}} \int_{\Omega}\left(\chi_{1}^{k+1} G_{\tau} * \chi_{2}^{k}+\chi_{2}^{k+1} G_{\tau} * \chi_{1}^{k}-\chi_{1}^{k+1} G_{\tau} * \chi_{2}^{k+1}\right) d \mathbf{x} \\
&+\int_{\Omega} \frac{\mu}{2}\left|D \mathbf{u}^{k}\right|^{2}-\mathbf{u}^{k} \cdot \mathbf{f} d \mathbf{x} .
\end{aligned}
$$

Then, we calculate

$$
\begin{aligned}
J^{\tau}\left(\chi^{k+1}, \mathbf{u}^{k}\right)-J^{\tau}\left(\chi^{k}, \mathbf{u}^{k}\right) & =\mathcal{L}_{\chi^{k}}^{\tau, k}\left(\chi^{k+1}\right)-\mathcal{L}_{\chi^{k}}^{\tau, k}\left(\chi^{k}\right)+\gamma \sqrt{\frac{\pi}{\tau}} \int_{\Omega}\left(\chi_{1}^{k+1}-\chi_{1}^{k}\right) G_{\tau} *\left(\chi_{2}^{k+1}-\chi_{2}^{k}\right) d \mathbf{x} \\
& =\mathcal{L}_{\chi^{k}}^{\tau, k}\left(\chi^{k+1}\right)-\mathcal{L}_{\chi^{k}}^{\tau, k}\left(\chi^{k}\right)-\gamma \sqrt{\frac{\pi}{\tau}} \int_{\Omega}\left(\chi_{1}^{k+1}-\chi_{1}^{k}\right) G_{\tau} *\left(\chi_{1}^{k+1}-\chi_{1}^{k}\right) d \mathbf{x} \\
& =\mathcal{L}_{\chi^{k}}^{\tau, k}\left(\chi^{k+1}\right)-\mathcal{L}_{\chi^{k}}^{\tau, k}\left(\chi^{k}\right)-\gamma \sqrt{\frac{\pi}{\tau}} \int_{\Omega}\left(G_{\tau / 2} *\left(\chi_{1}^{k+1}-\chi_{1}^{k}\right)\right)^{2} d \mathbf{x} \\
& \leq \mathcal{L}_{\chi^{k}}^{\tau, k}\left(\chi^{k+1}\right)-\mathcal{L}_{\chi^{k}}^{\tau, k}\left(\chi^{k}\right) .
\end{aligned}
$$

Because we have $\mathcal{L}_{\chi^{k}}^{\tau, k}\left(\chi^{k+1}\right)-\mathcal{L}_{\chi^{k}}^{\tau, k}\left(\chi^{k}\right) \leq 0$ from Lemma 3.3, we are led to

$$
J^{\tau}\left(\chi^{k+1}, \mathbf{u}^{k}\right)-J^{\tau}\left(\chi^{k}, \mathbf{u}^{k}\right) \leq 0
$$

for all $\tau>0$.

We are now led to the following theorem which proves the total energy decaying property

Theorem 4.1. For the series of minimizers

$$
\mathbf{u}^{0}, \chi^{1}, \mathbf{u}^{1}, \chi^{2}, \cdots, \mathbf{u}^{k}, \chi^{k+1}, \cdots,
$$

calculated with Algorithm 1, we have

$$
J^{\tau}\left(\chi^{k+1}, \mathbf{u}^{k+1}\right) \leq J^{\tau}\left(\chi^{k}, \mathbf{u}^{k}\right)
$$

for all $\tau>0$. 
Proof. For all $\tau>0$, from (3.7), we have

$$
J^{\tau}\left(\chi^{k+1}, \mathbf{u}^{k+1}\right) \leq J^{\tau}\left(\chi^{k+1}, \mathbf{u}^{k}\right) .
$$

From Lemma 4.1, we have

$$
J^{\tau}\left(\chi^{k+1}, \mathbf{u}^{k}\right) \leq J^{\tau}\left(\chi^{k}, \mathbf{u}^{k}\right)
$$

Thus, combining the above together gives the stability estimate (4.1).

Remark 4.1. We remark here that, as we proved, the energy is decaying for any given $\tau$. If $\tau$ changes from $\tau_{1}$ to $\tau_{2}$ at the $k^{\text {th }}$ iteration with $\tau_{1}>\tau_{2}$ in our adaptive in time strategy, for example, $\chi^{k}$ is generated by $\tau_{1}$ and $\chi^{k+1}$ is generated by $\tau_{2}$. The energy is decaying in the sense that $J^{\tau_{2}}\left(\chi^{k+1}, \mathbf{u}^{k+1}\right) \leq J^{\tau_{2}}\left(\chi^{k}, \mathbf{u}^{k}\right)$ where the energies $J$ at two iterations $\chi^{k}$ and $\chi^{k+1}$ are approximated by the same $\tau_{2}$.

\section{Numerical implementation}

In this section, we illustrate the implementation of Algorithm 1, with a focus on Step 2. The Brinkman equations (3.3a)-(3.3c) are solved with the mixed finite-element method, and the Taylor-Hood finite-element space is used for discretization, which satisfies the discrete inf-sup condition [18].

Let $\mathcal{T}_{h}$ be a uniform triangulation of the domain $\Omega$, and $\mathcal{N}_{h}$ is the set of all vertices of $\mathcal{T}_{h}$. For a given $\bar{\chi}_{h}=\left(\bar{\chi}_{1}^{h}, \bar{\chi}_{2}^{h}\right) \in \mathcal{B}_{h}$ where $\mathcal{B}_{h}$ is the discrete version of $\mathcal{B}$ defined on $\mathcal{N}_{h}$. For the uniform regular triangulation of the domain, all values are evaluated on uniform quad grid points. Thus, we can use FFT for efficient evaluation of the discretized convolutions.

We introduce the Taylor-Hood finite-element space

$$
\begin{aligned}
& V_{h}:=\left\{\mathbf{v} \in H^{1}\left(\Omega, \mathbb{R}^{d}\right)|\mathbf{v}|_{K} \in\left[P_{2}(K)\right]^{d}, K \in \mathcal{T}_{h}\right\}, \\
& Q_{h}:=\left\{q \in L^{2}(\Omega, \mathbb{R})\left|\int_{\Omega} q d \mathbf{x}=0, q\right|_{K} \in P_{1}(K), K \in \mathcal{T}_{h}\right\} .
\end{aligned}
$$

Let $V_{h}^{D}:=\left\{\mathbf{v} \in V_{h}|\mathbf{v}|_{\partial \Omega}=\mathbf{u}_{D}^{h}\right\}$, where $\mathbf{u}_{D}^{h}$ is a suitable approximation of the Dirichlet boundary condition $\mathbf{u}_{D}$ on the boundary edges/faces of $\mathcal{T}_{h}$. For the solution of (3.3a3.3c), find $\left(\mathbf{u}_{h}, p_{h}\right) \in V_{h}^{D} \times Q_{h}$ such that

$$
\begin{aligned}
-\left(p_{h}, \nabla \cdot \mathbf{v}_{h}\right)+\left(\mu \nabla \mathbf{u}_{h}, \nabla \mathbf{v}_{h}\right)+\left(\alpha\left(\bar{\chi}_{h}\right) \mathbf{u}_{h}, \mathbf{v}_{h}\right) & =\left(\mathbf{f}, \mathbf{v}_{h}\right), & & \forall \mathbf{v}_{h} \in V_{h}^{0}, \\
\left(\nabla \cdot \mathbf{u}_{h}, q_{h}\right) & =0, & & \forall q_{h} \in Q_{h} .
\end{aligned}
$$

The above bilinear form can be easily extended to the Brinkman equations both with Dirichlet boundary $\Gamma_{D}$ and Neumann boundary $\Gamma_{N}$, where $\Gamma_{D} \cap \Gamma_{N}=\varnothing, \Gamma_{D} \cup \Gamma_{N}=\partial \Omega$, and $\left.(\mu \nabla \mathbf{u}-p \boldsymbol{I}) \cdot \boldsymbol{n}\right|_{\Gamma_{N}}=g$. 
When $\mathbf{u}_{h}$ is obtained, we proceed to use the FFT to evaluate $\left(\phi_{1}^{h}, \phi_{2}^{h}\right)$ on each node of $\mathcal{N}_{h}$ as follows:

$$
\left\{\begin{array}{l}
\phi_{1}^{h}=\gamma \sqrt{\frac{\pi}{\tau}} G_{\tau} * \bar{\chi}_{2}^{h}, \\
\phi_{2}^{h}=\frac{\bar{\alpha}}{2} G_{\tau} *\left|\mathbf{u}_{h}\right|^{2}+\gamma \sqrt{\frac{\pi}{\tau}} G_{\tau} * \bar{\chi}_{1}^{h} .
\end{array}\right.
$$

Following Algorithm 1, we can now use $\left(\phi_{1}^{h}, \phi_{2}^{h}\right)$ to update the indicator function $\chi_{h}$ by the approach stated in Algorithm 1.

\section{Numerical experiments}

In this section, we perform extensive numerical testing to demonstrate the efficiency of Algorithm 1 with an adaptive strategy for the choice of $\tau=0.5$ as introduced in Remark 3.3.

\subsection{Two dimensional results}

We firstly test the proposed algorithm for the two dimensional problems. For most of examples in this subsection, we assume that the Dirichlet boundary condition with a parabolic profile and the magnitude of the velocity is set as $\left|\mathbf{u}_{D}\right|=\bar{g}\left(1-(2 t / l)^{2}\right)$ with $t \in[-l / 2, l / 2]$, where $l$ is the length of the section of the boundary at which the inflow/outflow velocity is imposed. The direction of the inflow/outflow velocity is illustrated in the following examples.

\section{Example 6.1}

The first example shown in Fig. 1 is the optimal design of a diffuser that was tested for topology optimization for fluids using MMA in [9]. Here, we apply Algorithm 3.2 to this

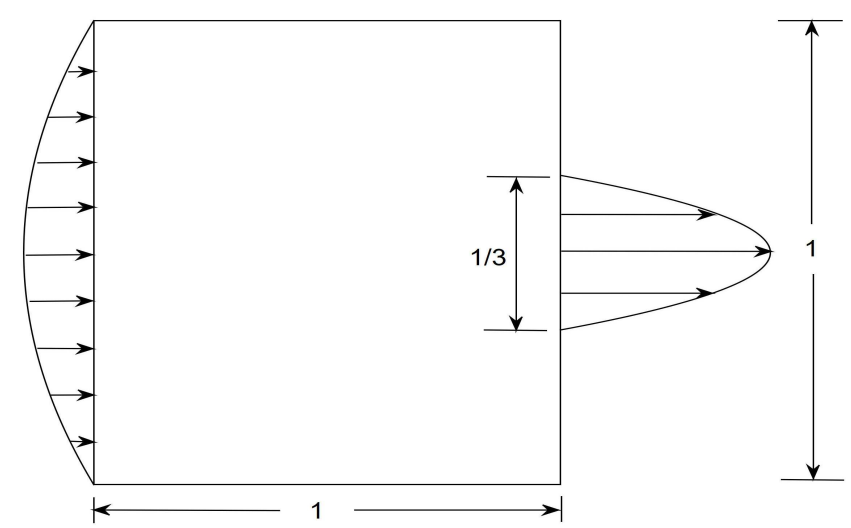

Figure 1: (Example 6.1) Design domain for the diffuser example. 

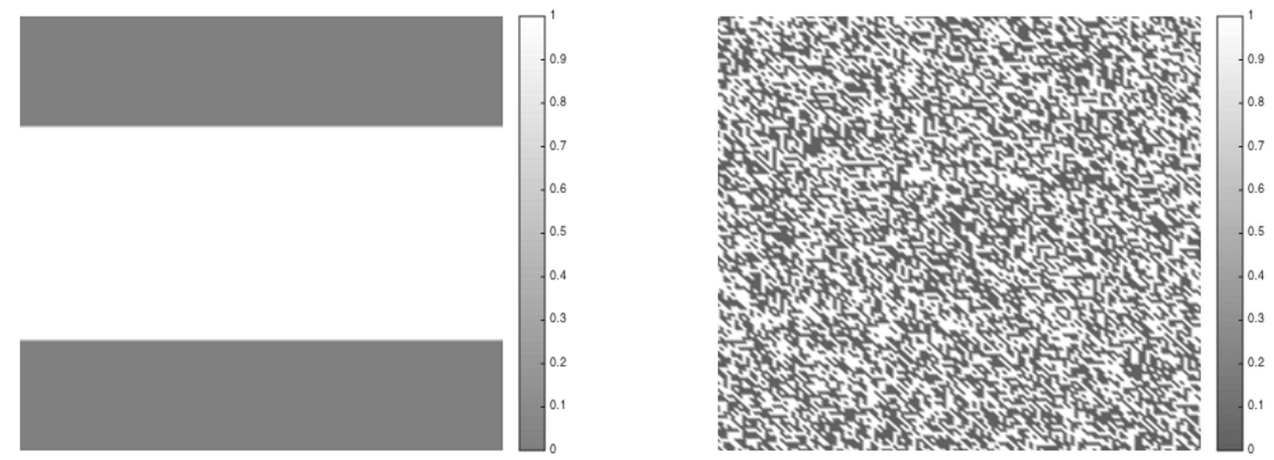

Figure 2: (Example 6.1) Left (Case 1): Initial distribution of $\chi_{1}$. Right (Case 2): Initial distribution of $\chi_{1}$.
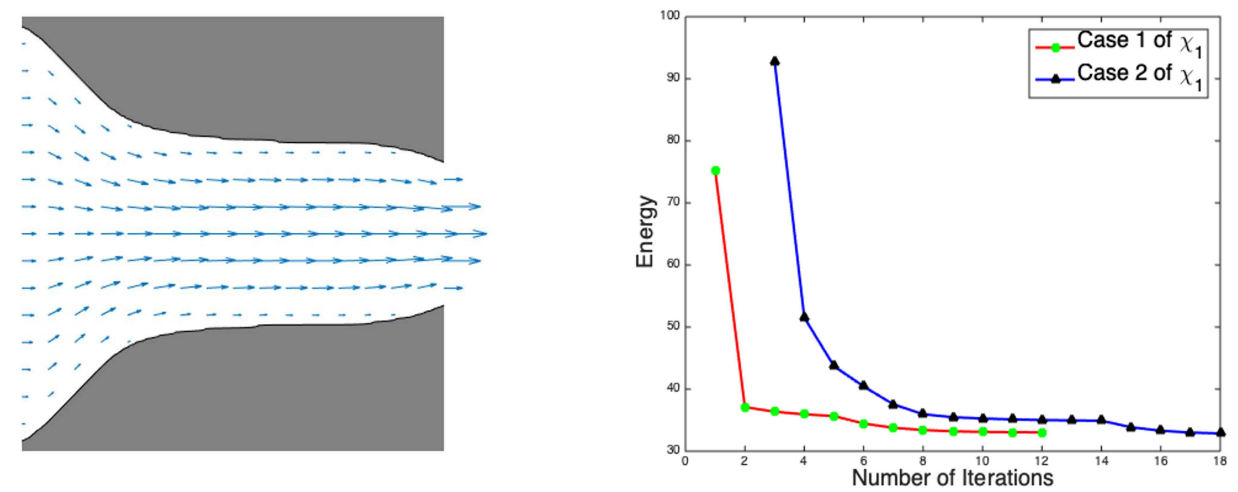

Figure 3: (Example 6.1) Left: Optimal diffuser for the case $\bar{\alpha}=2.5 \times 10^{4}$ and the approximate velocity in the fluid region. Right: Plot of energy curves for two cases of distribution of $\chi_{1}$. In this case, the parameters are set as $\bar{\alpha}=2.5 \times 10^{4}, \tau=0.001, \gamma=0.1$.

problem. Let $\bar{g}=1$ and 3 for the inflow and outflow velocities, respectively. We first set the fluid region fraction $\beta=0.5$ and using a $128 \times 128$ grid. We set the parameters $\bar{\alpha}=2.5 \times 10^{4}$, $\tau=0.001, \gamma=0.1$. We start with two different initial distributions of $\chi_{1}$, as shown in Fig. 2; that is, the initial fluid region is restricted in the middle of the domain in the left graph of Fig. 2 (Case 1), and the initial fluid region satisfies a random distribution in the right graph of Fig. 2 (Case 2). In both cases, we arrive at the same optimal design result shown in the left graph of Fig. 3, which also shows the quiver plot of the approximate velocity in the fluid region. The optimal design result is the same as the result obtained by MMA in [9]. The energy decaying property can be observed in the right graph of Fig. 3 which shows the energy curves for the above two cases of the initial distribution of $\chi_{1}$. The iteration converges in fewer than 18 steps in both cases.

In our algorithm, only a Brinkman problem is solved at each iteration and the indicator functions are easily updated based on simple convolutions followed by a thresholding 
Table 1: (Example 6.1) The CPU time (in seconds) in each iteration and the number of iterations.

\begin{tabular}{||c|c|c|c|c||}
\hline \multicolumn{2}{|c|}{ Grid } & $32 \times 32$ & $64 \times 64$ & $128 \times 128$ \\
\hline \hline \multirow{2}{*}{ Our algorithm } & Second/iter & 0.52 & 2.26 & 9.88 \\
\cline { 2 - 5 } & iter \# & 5 & 7 & 12 \\
\hline
\end{tabular}
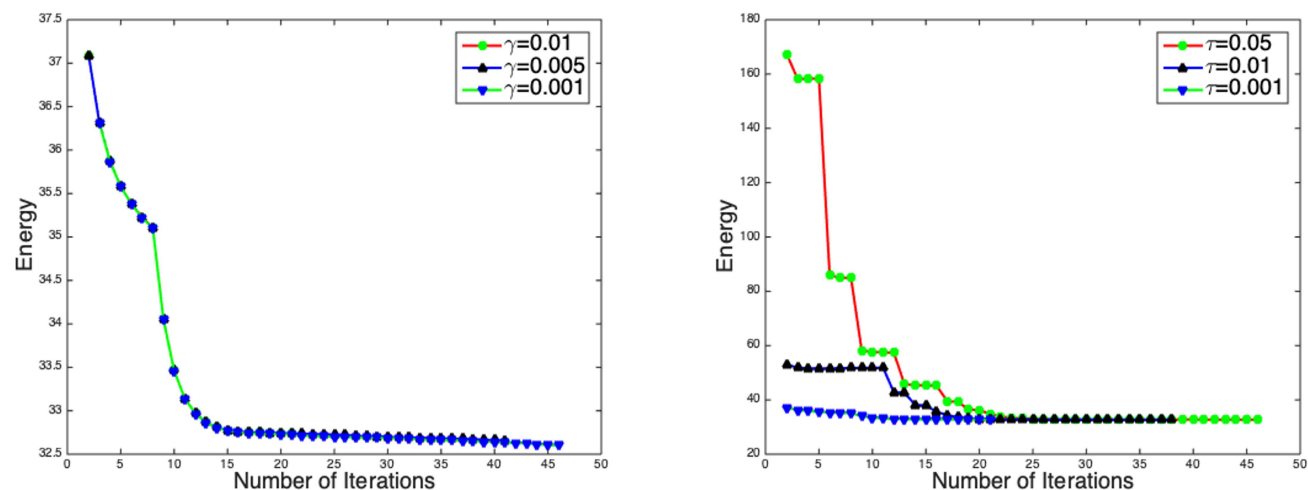

Figure 4: (Example 6.1) Plot of energy curves for case 1 of distribution of $\chi_{1}$ with $\bar{\alpha}=2.5 \times 10^{4}$. Left: For fixed $\tau=0.001$, energy curves for the cases of $\gamma=0.01,0.005,0.001$. Right: For fixed $\gamma=0.01$, energy curves for the cases of $\tau=0.05,0.01,0.001$.

step. Therefore, the computational cost at each iteration is optimal. What's more, Table 1 shows that the proposed algorithm only takes about 10 iterations before convergence for different discretizations of the computational domain, implying the efficiency and robustness of our algorithm.

Next, we test the case (initial fluid region of Case 1) for various parameters. We first fix $\bar{\alpha}=2.5 \times 10^{4}, \tau=0.001$ and vary $\gamma=0.01,0.005,0.001$. We then test the cases for fixed $\gamma=0.01$ and various choices of $\tau=0.05,0.01,0.001$. The optimal design of the diffuser is similar to the result in the left graph of Fig. 3. Fig. 4 shows the energy decaying property for each of these cases. In all cases, the iteration converges in fewer than 25 steps.

In the next example, we increase $\bar{\alpha}=2.5 \times 10^{5}$. Again, we use the initial fluid region of Case 1 with $\tau=0.001, \gamma=0.01$. The optimal design of the diffuser and the approximate velocity in the fluid region are shown in the left graph of Fig. 5. It seems that the fluid region at the left boundary reaches top and bottom boundaries in this case. The energy decaying property is also observed in Fig. 5. The iteration converges even more quickly at about 10 steps.

In order to further show the robustness of our algorithm, in the following we fix $\tau, \gamma$ and compare the numerical results of our algorithm with different choices of the parameter $\beta$. Here $\beta$ is related to the parameter $M$ in Algorithm 3.2. We test with the initial fluid region of Case 1 in Fig. 2. The optimal design of the diffuser (see Fig. 6) is obtained respectively for different choices of $\beta$, and we can see from Table 2 that our 

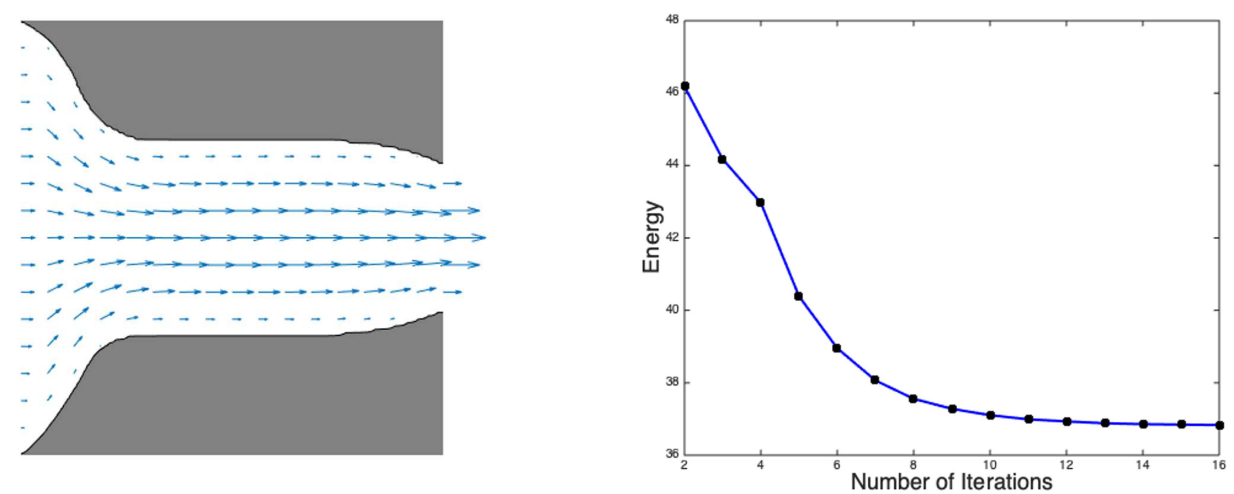

Figure 5: (Example 6.1) Left: Associated optimal diffuser and approximate velocity in the fluid region. Right: Plot of energy curve for Case 1 of distribution of $\chi_{1}$. In this case, the parameters are set as $\bar{\alpha}=2.5 \times 10^{5}$, $\tau=0.001, \gamma=0.01$.

Table 2: The number of iterations of our algorithm for Example 6.1 with $\bar{\alpha}=2.5 \times 10^{4}$ by different choices of $\beta$ and fixed $\tau=0.01$ and $\gamma=0.001$.

\begin{tabular}{||c|c||}
\hline$\beta$ & Number of iterations \\
\hline \hline $1 / 3$ & 27 \\
$2 / 5$ & 29 \\
$2 / 3$ & 27 \\
$3 / 4$ & 25 \\
\hline
\end{tabular}

algorithm always stably converges in fewer than 30 steps.

We also test the problem with the same inflow Dirichlet boundary condition as above, but we replace the outflow Dirichlet boundary condition with a homogeneous Neumann boundary. A similar optimal design of diffuser is then obtained as above for the cases of $\bar{\alpha}=2.5 \times 10^{4}$ and $\bar{\alpha}=2.5 \times 10^{5}$.

\section{Example 6.2}

In this example, we test the double pipes problem shown in Fig. 7. The inflow and outflow Dirichlet boundaries are located with centers $[0,1 / 4],[0,3 / 4],[1,1 / 4],[1,3 / 4]$, as shown in Fig. 7. Let $\bar{g}=1$ for the inflow and outflow velocities, respectively, and let the fluid region fraction be $\beta=1 / 3$. We test the problem with $\bar{\alpha}=2.5 \times 10^{4}$ on a $128 \times 256$ grid for $d=0.5$ and on a $192 \times 128$ grid for $d=1.5$.

For the case $d=0.5$, we choose a random initial distribution $\chi_{1}$, as shown in the left graph of Fig. 8. We remark that $\gamma$ can also be set to zero in Algorithm 1. For fixed $\tau=0.001$, we test $\gamma=0.01,0.001,0$. The optimal design result is nearly the same for the three choices of $\gamma$, as shown in the middle graph of Fig. 8, and the energy decaying property is observed from the energy curves in the right graph of Fig. 8. 

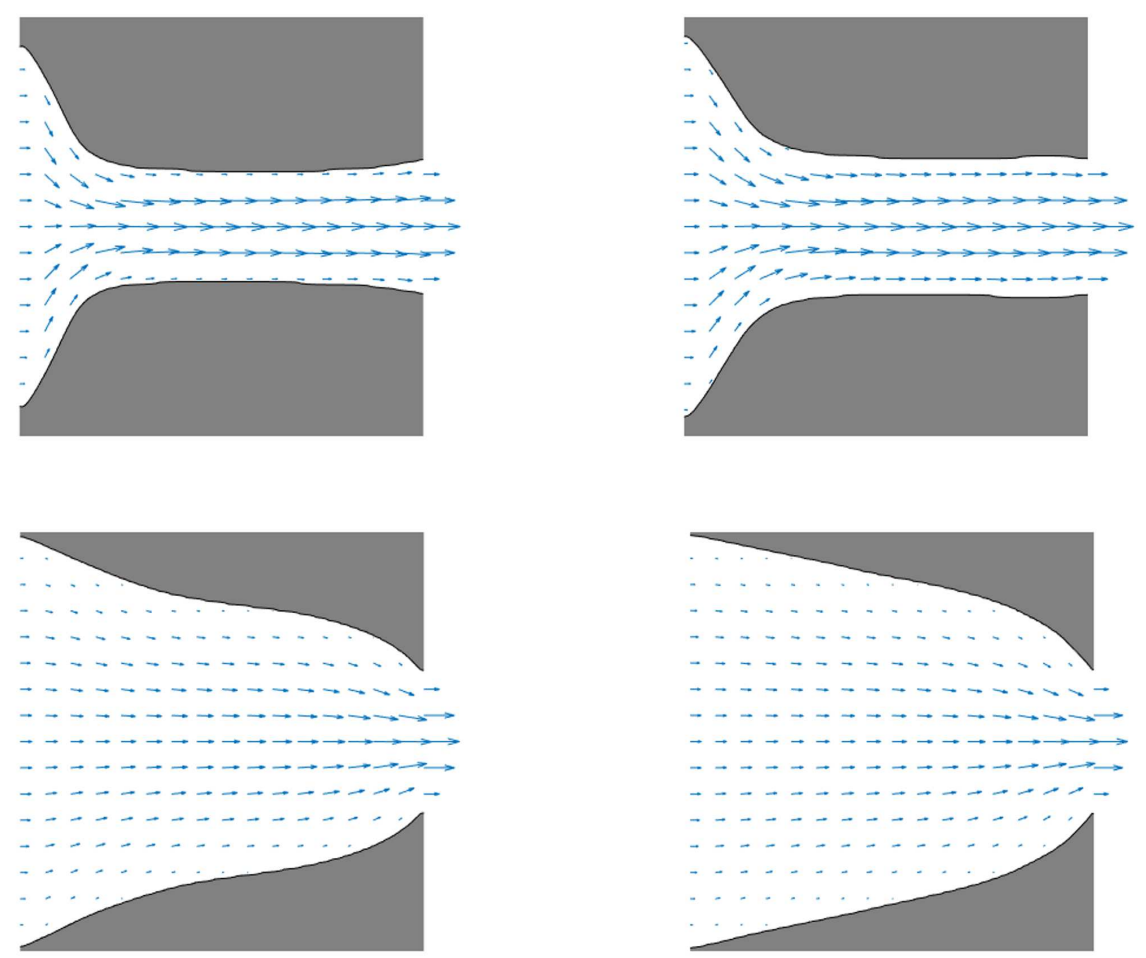

Figure 6: (Example 6.1) Associated optimal diffuser and approximate velocity in the fluid region based on different choices $\beta$. Top-left: $\beta=1 / 3$; Top-right: $\beta=2 / 5$; Bottom-left: $\beta=2 / 3$; Bottom-right: $\beta=3 / 4$. In this case, we fix the parameters as $\bar{\alpha}=2.5 \times 10^{4}, \tau=0.01, \gamma=0.001$.

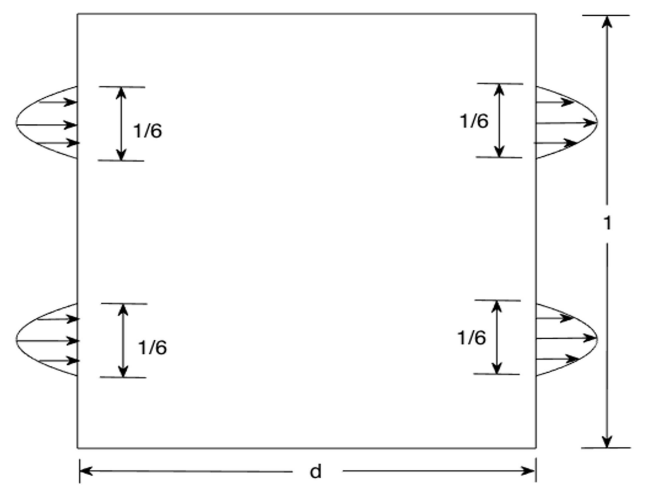

Figure 7: (Example 6.2) Design domain for the double pipes example.

For the case $d=1.5$, we choose an initial distribution $\chi_{1}$ with the fluid region located in the middle of the domain as Case 1 of Example 6.1. We set $\tau=0.01$ and $\gamma=0.0001$. The optimal design result and the approximate velocity are shown in the left graph of Fig. 9, 

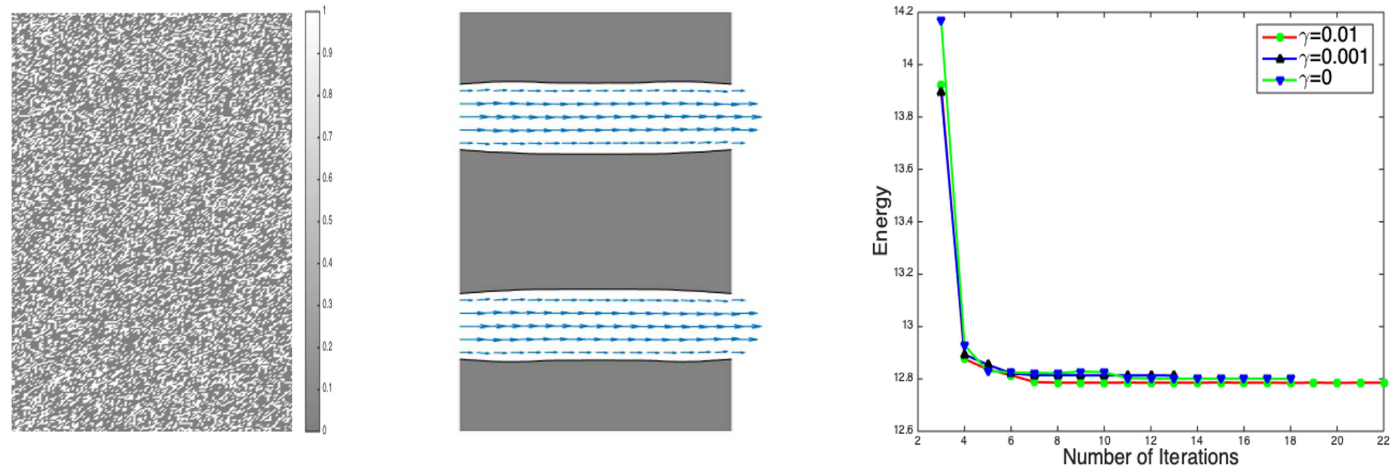

Figure 8: (Example 6.2) For the case $d=0.5$. Left: Initial distribution of $\chi_{1}$. Middle: Optimal double pipes and approximate velocity in the fluid region. Right: For fixed $\tau=0.001$, energy curves for the cases of $\gamma=0.01,0.001,0$.
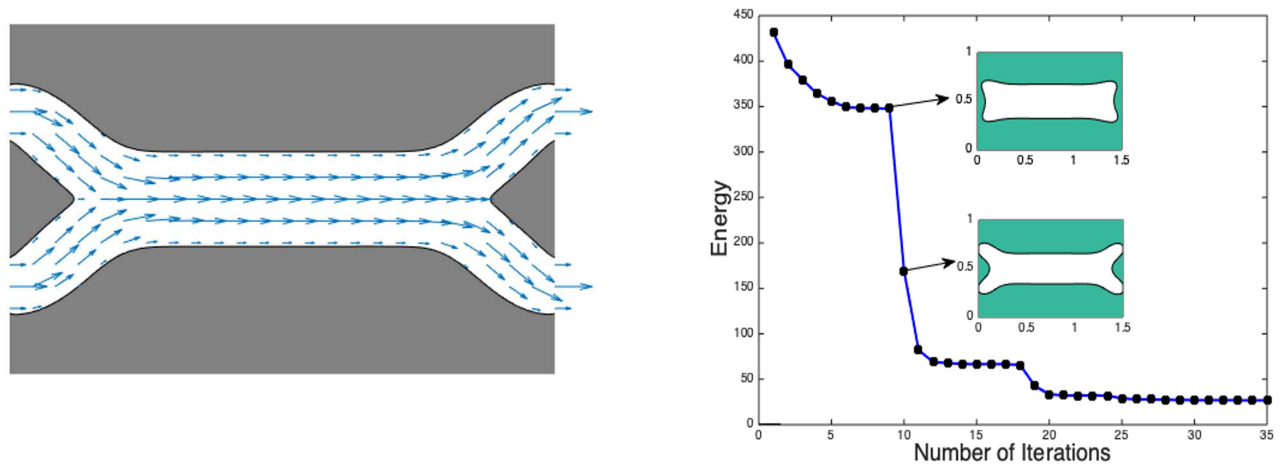

Figure 9: (Example 6.2) For the case $d=1.5$, the parameters are set as $\tau=0.01$ and $\gamma=0.0001$. Left: Optimal double pipes and approximate velocity in fluid region. Right: Energy curve.

and the energy decaying property is also observed from the energy curve in the right graph of Fig. 9. For the large jump in this energy curve at the iteration steps 9 and 10, we observe that this is due to the fact that there is a large topology change of the fluid and solid regions at these two steps. Compared with the computational cost used by MMA in [9], we find that our algorithm converges more quickly to the optimal result.

\section{Example 6.3}

We consider another example studied in [9] that includes a body fluid force term imposed in the local circular region with center $[1 / 2,1 / 3]$ and radius $r=1 / 12$. We show the design domain in Fig. 10. The inflow and outflow Dirichlet boundaries are located with centers $[0,2 / 3]$ and $[1,2 / 3]$ respectively. Let $\bar{g}=1$ for the inflow and outflow velocities, and let the fluid region fraction be $\beta=1 / 4$. We test the problem with various choices of body 


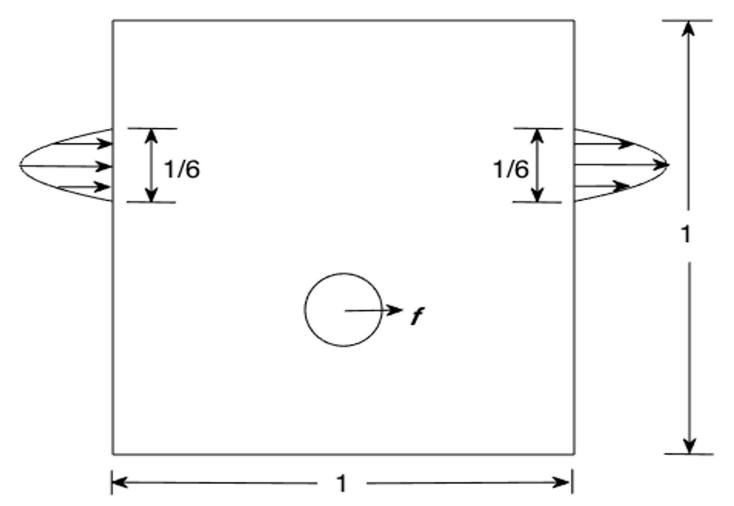

Figure 10: (Example 6.3) Design domain for the example with a force term.
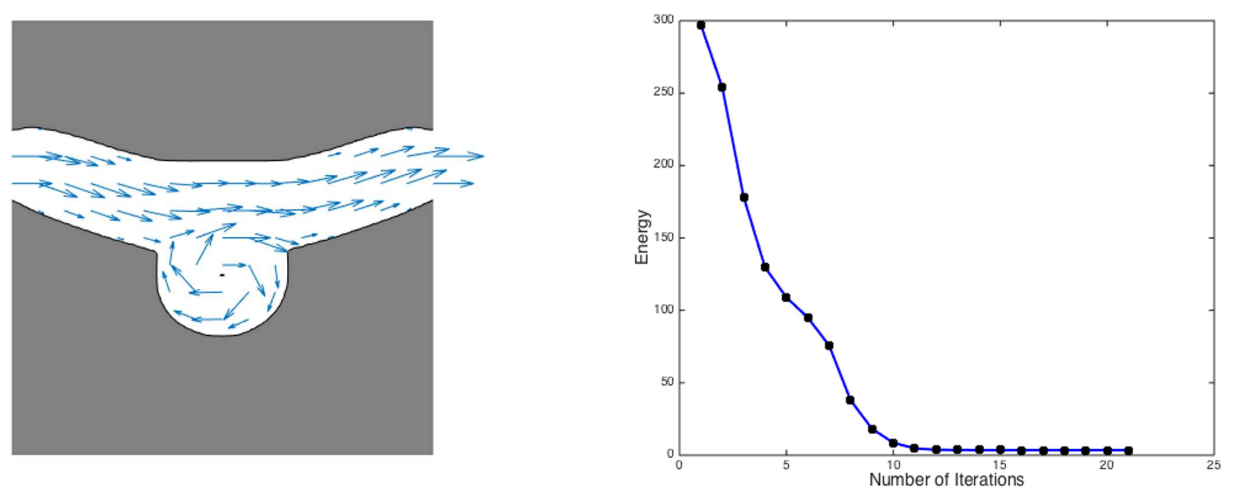

Figure 11: (Example 6.3) For the example with a force term $\mathbf{f}=[-1125,0]$ on a grid $128 \times 128$. Left: Optimal design result and approximate velocity in the fluid region. Right: Energy curve.

fluid force on a $128 \times 128$ grid, and we always choose $\bar{\alpha}=2.5 \times 10^{4}, \tau=0.01, \gamma=0.0001$ in this example.

We test the cases for three different force terms $\mathbf{f}=[-1125,0],[562.5,0],[1687.5,0]$. We choose the initial distribution $\chi_{1}$ with the fluid region located in a circular region with center $[1 / 2,1 / 2]$ and radius $1 / \sqrt{3 \pi}$. The optimal results and energy curves are plotted in Figs. 11 to 13 for various values for force $\mathbf{f}$, and the new algorithm also converges more quickly to the optimal results than the MMA shown in [9]. One can observe that for $\mathbf{f}=[-1125,0]$ the fluid flow is in a clockwise direction near the center roundabout (left graphs in Fig. 11), while for $\mathbf{f}=[1687.5,0]$ it is in a counterclockwise direction (left graph of Fig. 13).

An interesting phenomenon observed in this example was the appearance of a tiny local solid at the center of the roundabout for the two cases of $\mathbf{f}=[-1125,0],[1687.5,0]$, and the tiny local solid is clearer when the grid is finer (cf. Fig. 14). 

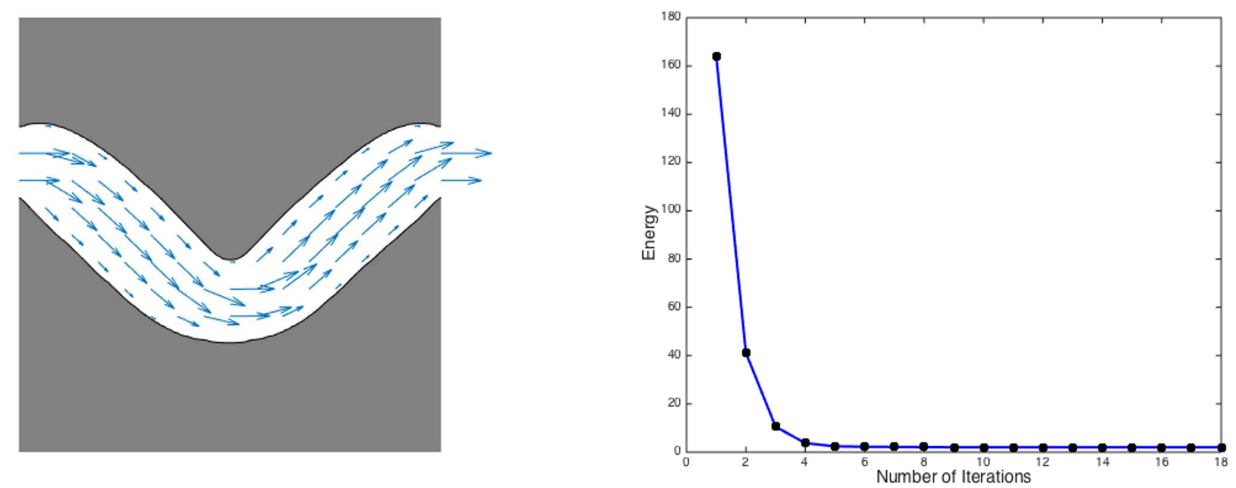

Figure 12: (Example 6.3) For the example with a force term $[562.5,0]$ on a grid $128 \times 128$. Left: Optimal design result and approximate velocity in the fluid region. Right: Energy curve.
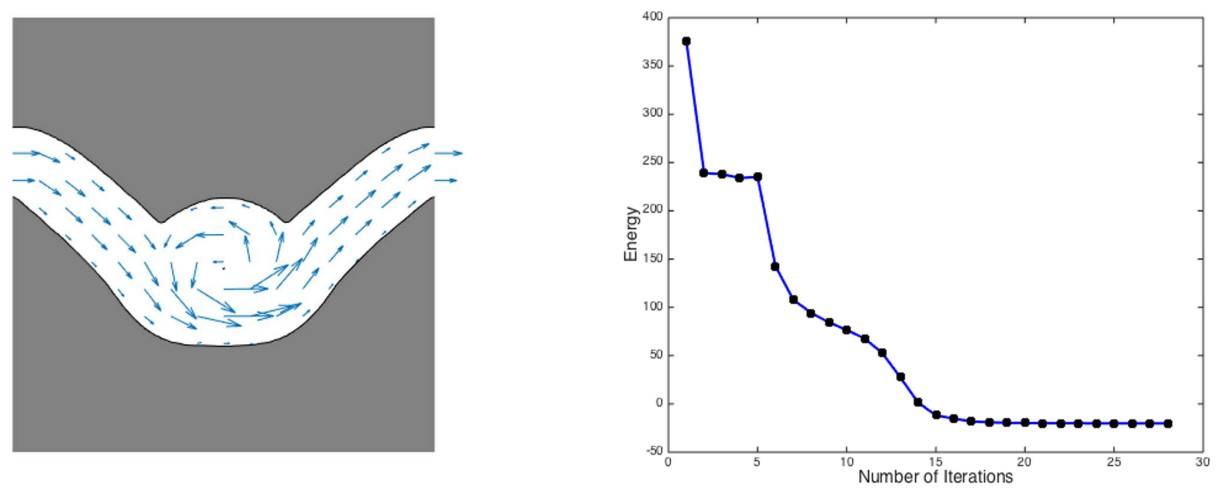

Figure 13: (Example 6.3) For the example with a force term $[1687.5,0]$ on a grid $128 \times 128$. Left: Optimal design result and approximate velocity in the fluid region. Right: Energy curve.
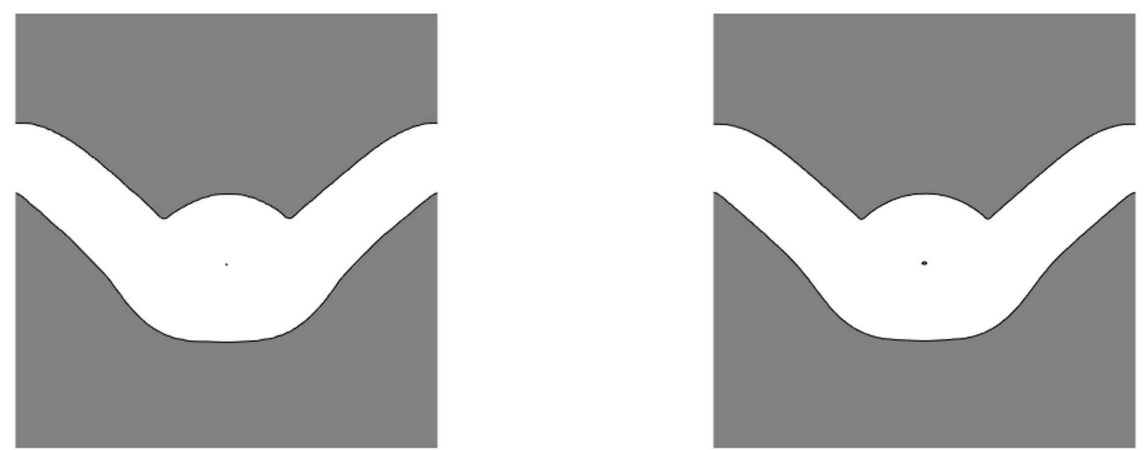

Figure 14: (Example 6.3) Optimal design results for example with force term $\mathbf{f}=[1687.5,0]$. Left: Optimal design result on a coarse grid $128 \times 128$. Right: Optimal design result on a fine grid $256 \times 256$. 


\section{Example 6.4}

Finally, we consider optimal design for a three-terminal device shown in Fig. 15. The inflow and outflow Dirichlet boundaries are located with centers $[0,0.3]$ and $[1,0.7]$, and the homogeneous Neumann boundary is located on the left boundary with center $[0,1.1]$. Let $\bar{g}=0.5$ for the inflow velocity and the fluid region fraction be $\beta=0.3$. We choose $\bar{\alpha}=2.5 \times 10^{4}, \tau=0.01, \gamma=0.0001$ in this example and test the problem on a grid $80 \times 112$.

We choose the initial distribution $\chi_{1}$, with the fluid region located in double parallel pipes $[0,1] \times[13 / 60,23 / 60] \cup[0,1] \times[37 / 60,47 / 60]$. The optimal result was obtained after 29 iterations. The optimal design result and the approximate velocity are shown in the left graph of Fig. 16. The energy decaying property is also observed from the energy curve in the right graph of Fig. 16.

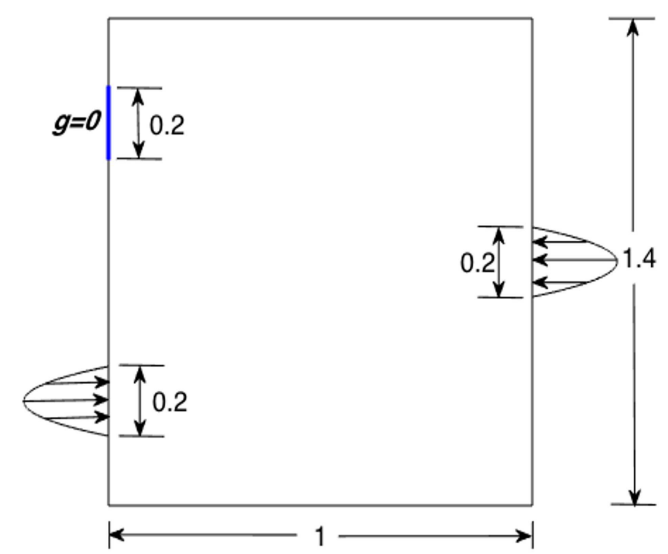

Figure 15: (Example 6.4) Design domain for the example with a force term.
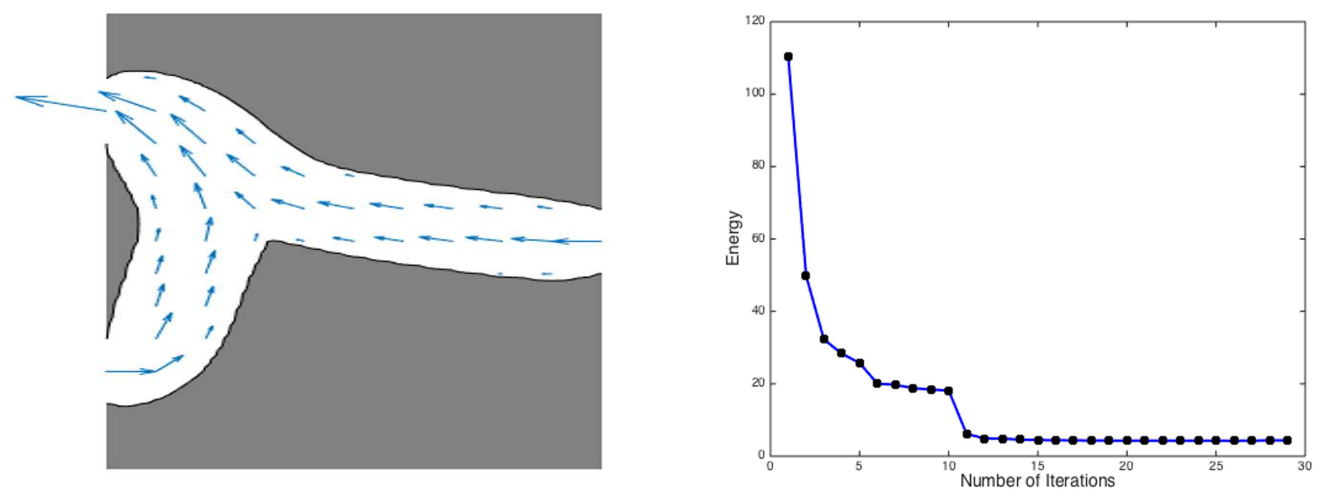

Figure 16: (Example 6.4) Left: Optimal design result for example of three-terminal device and approximate velocity in the fluid region. Right: Energy curve. 


\subsection{Three dimensional results}

We now present the numerical examples in three dimensions. For the Dirichlet boundary condition in the following examples, we always assume that the magnitude of the velocity is set as

$$
\left|\mathbf{u}_{D}\right|=\bar{g}\left(1-\frac{(y-a)^{2}+(z-b)^{2}}{l^{2}}\right),
$$

where $\bar{g}$ is the prescribed velocity at the center of the flow profile at which the inflow/outflow velocity is imposed, $l$ is the radius of the flow profile, $(y, z)$ are Cartesian coordinates on a $x$-plane, and $(a, b)$ are the center of a circle on a $x$-plane.

\section{Example 6.5}

The design domain of this example is shown in Fig. 17. For the inflow, we let $\bar{g}=1$, $l=\frac{1}{2}$, and $(a, b)=\left(\frac{1}{2}, \frac{1}{2}\right)$ on $x=0$ plane. For the objective of mass conservation, we let $\bar{g}=9, l=\frac{1}{6}$, and $(a, b)=\left(\frac{1}{2}, \frac{1}{2}\right)$ on $x=1$ plane. We set the fluid region fraction is $\beta=0.35$. This example was already tested by the level set method in [10]. Here we apply our new Algorithm 3.2 to obtain the optimal diffuser. Throughout this example, we choose the initial distribution $\chi_{1}$ with fluid domain in a region of $\{(x, y, z): x \in(0,1), y \in(0,1), z \in$ $\left.\left(\frac{7}{20}, \frac{7}{10}\right)\right\}$.

Firstly, we test the case with $\bar{\alpha}=2.5 \times 10^{4}, \tau=0.05$, and $\gamma=0.01$ on $32 \times 32 \times 32$ and $64 \times 64 \times 64$ grids. In the following, the interface between solid and fluid regions for the optimal design is shown, and the fluid region locates in the interior of subdomain surrounded by the interface. The optimal diffusers are presented in the left graphs of Fig. 18

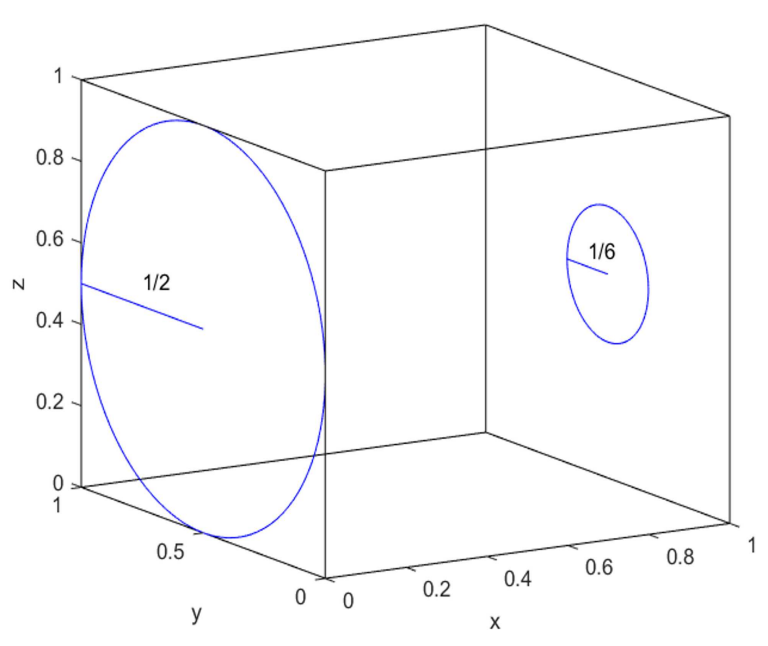

Figure 17: (Example 6.5) Design domain. 

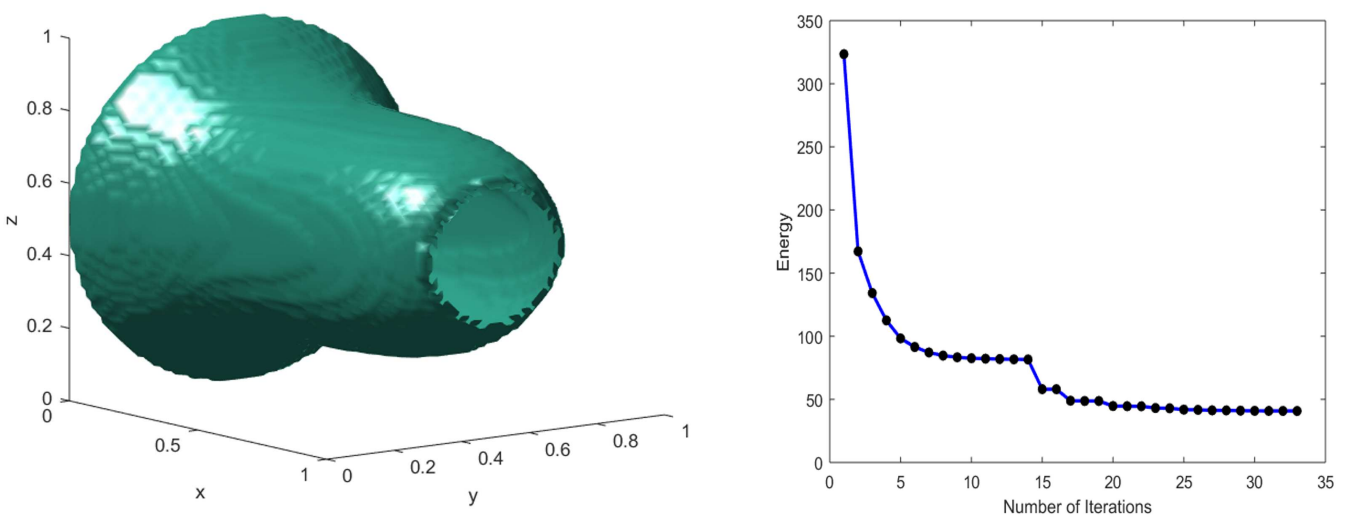

Figure 18: (Example 6.5) Left: Optimal design result on a $32 \times 32 \times 32$ grid. Right: Energy curve. In this case the parameters are set as $\bar{\alpha}=2.5 \times 10^{4}, \tau=0.05, \gamma=0.01$.
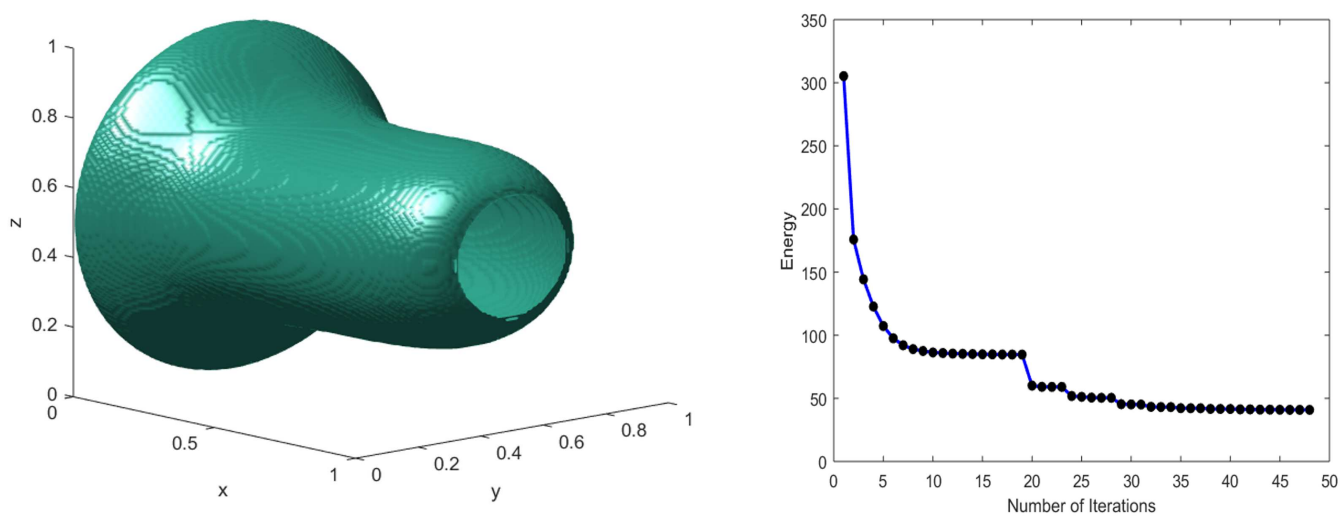

Figure 19: (Example 6.5) Left: Optimal design result on a $64 \times 64 \times 64$ grid. Right: Energy curve. In this case the parameters are set as $\bar{\alpha}=2.5 \times 10^{4}, \tau=0.05, \gamma=0.01$.

and Fig. 19 and the energy decaying property can be observed in the right graphs of Fig. 18 and Fig. 19. The optimal design results seem to be similar to that in [10]. The iteration converges in about 25 steps and 35 steps on coarse and fine grids respectively. Additionally, the slice of optimal design result at $y=0.5$ on $32 \times 32 \times 32$ grid and the approximate velocity in the fluid domain are provided in Fig. 20.

Next, the energy decay properties of the Algorithm 3.2 with different parameters $\tau$ and $\gamma$ for this problem are shown for the same case of $\bar{\alpha}=2.5 \times 10^{4}$ in Fig. 21. We note that the optimal design results for different parameters $\tau$ and $\gamma$ are similar to that in the left graphs of Fig. 18 and Fig. 19. From the two graphs of Fig. 21, we find that the energy converges to almost the same value when $\tau$ or $\gamma$ is fixed. 


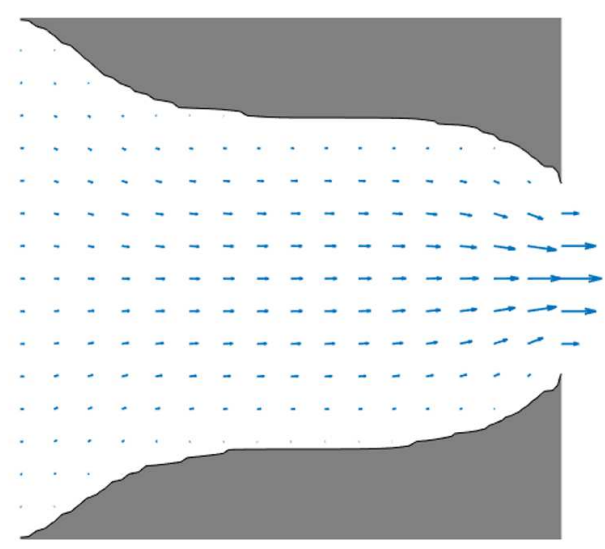

Figure 20: (Example 6.5) The slice of optimal design result and the approximate velocity in fluid region at $y=0.5$ on a $32 \times 32 \times 32$ grid. The parameters are set as $\bar{\alpha}=2.5 \times 10^{4}, \tau=0.05, \gamma=0.01$.
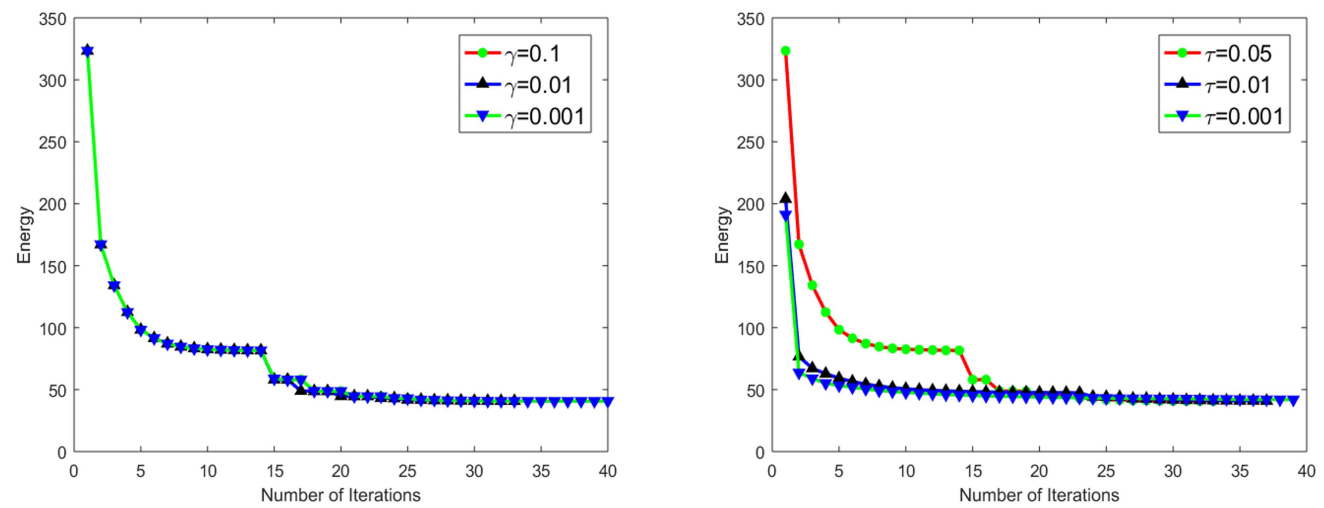

Figure 21: (Example 6.5) Plot of energy curves for $\bar{\alpha}=2.5 \times 10^{4}$ on $32 \times 32 \times 32$ grid. Left: For fixed $\tau=0.05$, energy curves for the cases of $\gamma=0.1,0.01,0.001$. Right: For fixed $\gamma=0.01$, energy curves for the cases of $\tau=0.05,0.01,0.001$.

\section{Example 6.6}

In this example we assume that there are four flow profiles on the inflow boundary and one flow profile on the outflow boundary. The design domain is shown in Fig. 22. For the four inflow profiles, we let $\bar{g}=1$, the radius is set as $l=\frac{1}{8}$ and the centers of circles are $\left(\frac{1}{4}, \frac{1}{4}\right),\left(\frac{1}{4}, \frac{3}{4}\right),\left(\frac{3}{4}, \frac{1}{4}\right)$ and $\left(\frac{3}{4}, \frac{3}{4}\right)$ on the $x=0$ plane respectively. For the outflow profile, we let $\bar{g}=1, l=\frac{1}{4}$ and $(a, b)=\left(\frac{1}{2}, \frac{1}{2}\right)$ on the $x=1$ plane. We set the fluid region fraction as $\beta=\frac{1}{4}$.

We test this problem based on the Algorithm 3.2 with $\bar{\alpha}=2.5 \times 10^{4}, \tau=0.05$, and $\gamma=0.01$ on $32 \times 32 \times 32$ and $64 \times 64 \times 64$ grids. The initial distribution $\chi_{1}$ with fluid domain is located in a region of $\left\{(x, y, z): x \in(0,1), y \in(0,1), z \in\left(\frac{1}{2}, \frac{3}{4}\right)\right\}$. The corresponding optimal 


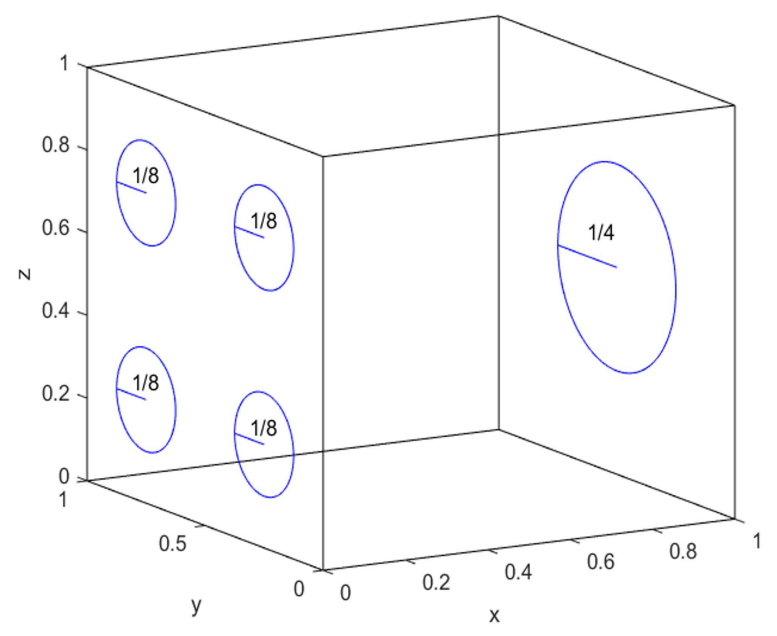

Figure 22: (Example 6.6) Design domain.
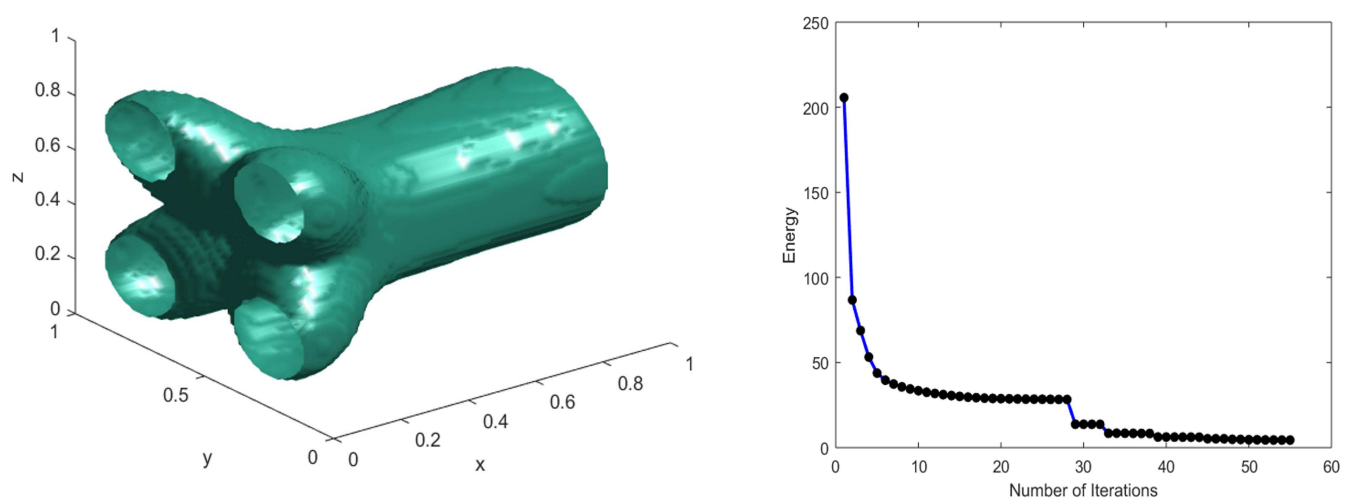

Figure 23: (Example 6.6) Left: Optimal design result on a $32 \times 32 \times 32$ grid. Right: Energy curve. In this case the parameters are set as $\bar{\alpha}=2.5 \times 10^{4}, \tau=0.05, \gamma=0.01$.

design result is shown in the left graphs of Fig. 23 and Fig. 24. From the left graphs of Fig. 23 and Fig. 24, we can see that the interface between solid and fluid regions is more smooth when the simulation is performed on the fine grid. From the right graphs of Fig. 23 and Fig. 24, the energy decaying property is also observed. The iteration converges in about 50 steps and 70 steps on coarse and fine grids respectively. In Fig. 25, we present the slice of optimal design result at $z=25 / 64$ on a $32 \times 32 \times 32$ grid, and the approximate velocity in the fluid region is also included. 

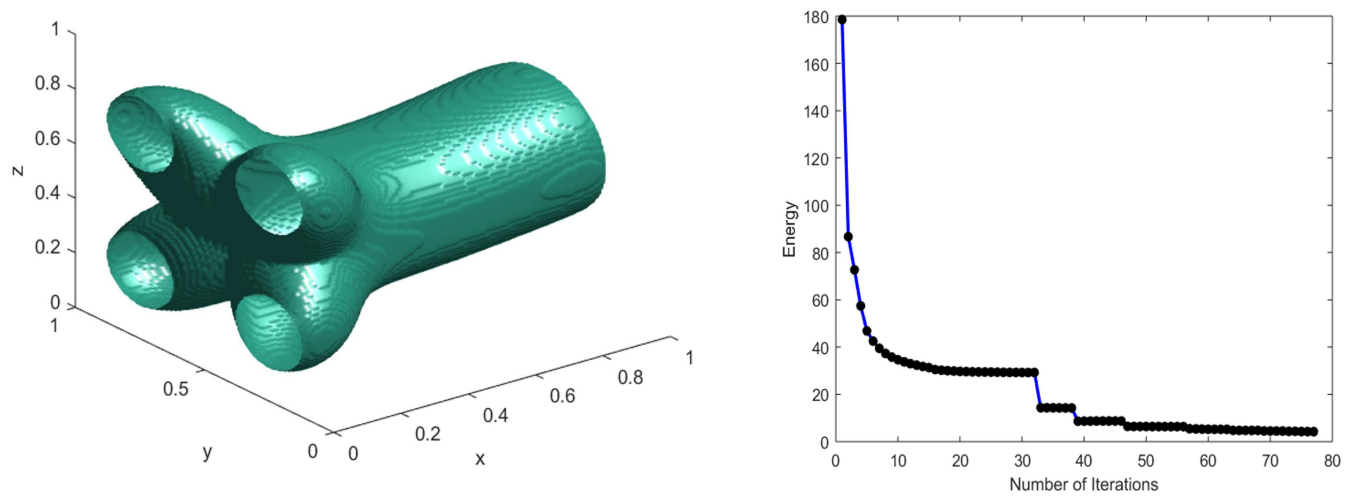

Figure 24: (Example 6.6) Left: Optimal design result on a $64 \times 64 \times 64$ grid. Right: Energy curve. In this case the parameters are set as $\bar{\alpha}=2.5 \times 10^{4}, \tau=0.05, \gamma=0.01$.

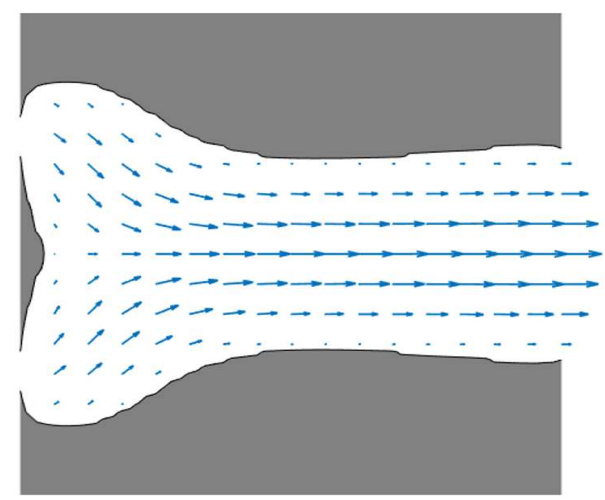

Figure 25: (Example 6.6) The slice of optimal design result and the approximate velocity in fluid region at $z=25 / 64$ on a $32 \times 32 \times 32$ grid. The parameters are set as $\bar{\alpha}=2.5 \times 10^{4}, \tau=0.05, \gamma=0.01$.

\section{Discussion and conclusions}

In this paper, we introduce a new efficient threshold dynamics method for topology optimization for fluids in Stokes flow. We aim to minimize a total energy functional that consists of the dissipation power and the perimeter approximated by nonlocal energy. During the iterations of the algorithm, only a Brinkman equation requires solution by a mixed finite-element method, and the indicator functions of fluid-solid regions are updated by a thresholding step that is based on the convolutions computed by the FFT. A simple adaptive in time strategy is used to accelerate the convergence of the algorithm. The total energy decaying property of the proposed algorithm is rigorously proved and 
observed numerically. Several numerical examples are presented to verify the efficiency of the new algorithm, and we show that the new algorithm converges faster than some of the existing methods for topology optimization for fluids. For the numerical experiments that we have performed thus far, the numerical results are robust and relatively insensitive to initial guesses and parameters.

We have been working on extending the threshold dynamics method to topology optimization for Navier-Stokes flow or more complicated multi-physics related problems. We notice that the proof of the energy decaying of the scheme in the current paper is due to the fact that the Brinkman equation (the constraint in the optimization problem) is the exact variation of the objective functional which may not be the case in general. Also for problems with many possible local minimizers, the iteration may stuck in local minimizers. Therefore, careful choice of initial guesses is necessary. These issues will be carefully investigated and reported elsewhere.

\section{Acknowledgments}

This research was supported in part by the Hong Kong Research Grants Council (GRF grants 16308421, 16305819, 16303318) and CUHKSZ-UDF01002028. H. Chen and D. Wang acknowledge the hospitality of the Department of Mathematics at the Hong Kong University of Science and Technology during their visit. The work of Huangxin Chen was supported by the NSF of China (Grant No. 11771363, 12122115). D. Wang acknowledges support from the NSF of China (Grant No. 12101524) and CUHKSZ-UDF01001803.

\section{References}

[1] R. Adams, Sobolev Spaces, Academic Press, New York, 1975.

[2] G. Allaire, F. Jouve, and A. M. Toader, Structural optimization using sensitivity analysis and a level-set method, Journal of Computational Physics, 194(1), 363-393, 2004.

[3] G. Allaire, C. Dapogny, G. Delgado, and G. Michailidis, Multi-phase structural optimization via a level set method, ESAIM: Control, Optimisation and Calculus Of Variations, 20(2), 576-611, 2014.

[4] G. Allaire, Optimal design for minimum weight and compliance in plane stress using extrernal microstructures, Eur. J. Mech. A/Solids 12, 839-878, 1993.

[5] G. Allaire and F. Jouve, A level-set method for vibration and multiple loads structural optimization, Computer Methods in Applied Mechanics And Engineering, 194(30-33), 32693290, 2005.

[6] C. S. Andreasen, A. R. Gersborg, and O. Sigmund, Topology optimization of microfluidic mixers, Int. J. Numer. Methods Fluids, 61, 498-513, 2009.

[7] M. P. Bendsøe. Optimal shape design as a material distribution problem. Structural Optimization, 1(4), 193-202, 1989.

[8] M. P. Bendsøe and O. Sigmund, Topology Optimization: Theory, Methods and Applications, Springer, 2003.

[9] T. Borrvall and J. Petersson, Topology optimization of fluids in Stokes flow, Int. J. Numer. Methods Fluids, 41, 77-107, 2003. 
[10] V. J. Challis and J. K. Guest, Level set topology optimization of fluids in Stokes flow, Int. J. Numer. Meth. Engrg., 79, 1284-1308, 2009.

[11] H. Chen and X. P. Wang, A one-domain approach for modeling and simulation of free fluid over a porous medium, J. Comput. Phys., 259, 650-671, 2014.

[12] M. Elsey and S. Esedoḡlu, Threshold dynamics for anisotropic surface energies, Math. Comp., 87, 1721-1756, 2018.

[13] S. Esedoğlu and F. Otto, Threshold dynamics for networks with arbitrary surface tensions, Comm. Pure Appl. Math., 68, 808-864, 2015.

[14] S. Esedoḡlu, S. J. Ruuth, and R. Tsai, Threshold dynamics for high order geometric motions, Interfaces and Free Boundaries, 10, 263-282, 2008.

[15] S. Esedoğlu, Y. H. R. Tsai, Threshold dynamics for the piecewise constant Mumford-Shah functional, J. Comput. Phys., 211, 367-384, 2006.

[16] L. C. Evans, Convergence of an algorithm for mean curvature motion, Indiana University Mathematics Journal, 42, 533-557, 1993.

[17] H. Garcke, C. Hecht, M. Hinze, and C. Kahle, Numerical approximation of phase field-based shape and topology optimization for fluids, SIAM J. Sci. Comput., 37, A1846-A1871, 2015.

[18] V. Girault and P. Raviart, Finite Element Methods for Navier-Stokes Equations: Theory and Algorithms, Springer-Verlag, Berlin, Heidelberg, New York, 1986.

[19] E. Giusti, Minimal Surfaces and Functions of Bounded Variation, Birkhäuser, Boston, 1984.

[20] K. Ishii, Optimal rate of convergence of the Bence-Merriman-Osher Algorithm for motion by mean curvature, SIAM J. Math. Anal., 37, 841-866, 2005.

[21] M. Jacobs, E. Merkurjev, and S. Esedoḡlu, Auction dynamics: A volume constrained MBO scheme, J. Comput. Phys., 354, 288-310, 2018.

[22] N. Jenkins and K. Maute, Level set topology optimization of stationary fluid-structure interaction problems. Structural and Multidisciplinary Optimization, 52(1), 179-195, 2015.

[23] S. Jiang, D. Wang, and X. P. Wang, An efficient boundary integral scheme for the MBO threshold dynamics method via NUFFT, J. Sci. Comput., 74, 474-490, 2018.

[24] S. $\mathrm{Lu}$ and $\mathrm{X} . \mathrm{Xu}$, An efficient diffusion generated motion method for wetting dynamics, submitted, 2021.

[25] J. Ma, D. Wang, X.P. Wang, and X. Yang, A fast algorithm for geodesic active contours with applications to medical image segmentation. arXiv preprint arXiv:2007.00525, 2020.

[26] B. Merriman, J. K. Bence, and S. Osher, Diffusion generated motion by mean curvature, UCLA CAM Report 92-18, 1992.

[27] A. A. Novotny and J. Soko lowski, Topological Derivatives in Shape Optimization, Springer Science \& Business, Media, 2012.

[28] F. Okkels, L. H. Olesen, and H. Bruus, Application of topology optimization in the design of micro and nanofluidic systems, NSTI-Nanotech, 575-578, 2005.

[29] F. Okkels, H. Bruus, Scaling behavior of optimally structured catalytic microfluidic reactors, Phys. Rev. E, 75, 1-4, 2007.

[30] S. Osher and J. A. Sethian, Fronts propagating with curvature-dependent speed: algorithms based on Hamilton-Jacobi formulations. Journal of Computational Physics, 79(1), 12-49, 1988.

[31] B. Osting and D. Wang, A diffusion generated motion for orthogonal matrix-valued fields, Math. Comp., 89, 515-550, 2020.

[32] B. Osting and D. Wang, Diffusion generated methods for denoising target-valued images, AIMS Inverse Probl. Imag., 14(2), 205-232, 2020.

[33] G. Pingen and K. Maute, Optimal design for non-Newtonian flows using a topology opti- 
mization approach, Comput. Math. Appl., 59, 2340-2350, 2010.

[34] A. Takezawa, S. Nishiwaki, and M. Kitamura, Shape and topology optimization based on the phase field method and sensitivity analysis, J. Comput. Phys, 229, 2697-2718, 2010.

[35] S. J. Ruuth, A diffusion-generated approach to multiphase motion, J. Comput. Phys., 145, 166-192, 1998.

[36] S. J. Ruuth and B. T. R. Wetton, A simple scheme for volume-preserving motion by mean curvature, J. Sci. Comput., 19, 373-384, 2003.

[37] K. Svanberg, The method of moving asymptotes-a new method for structural optimization, Int. J. Numer. Meth. Engrg., 24, pp. 359-373, 1987.

[38] Swartz, D. and Yip, N.K., Convergence of diffusion generated motion to motion by mean curvature. Communications in Partial Differential Equations, 42(10), 1598-1643, 2017.

[39] C. H. Villanueva and K. Maute, CutFEM topology optimization of 3D laminar incompressible flow problems, Comput. Methods Appl. Mech. Engrg., 320, 444-473, 2017.

[40] D. Wang, An efficient iterative method for reconstructing surface from point clouds. Journal of Scientific Computing, 87(1), 1-21, 2021.

[41] D. Wang, S. Jiang, and X.P. Wang, An efficient boundary integral scheme for the threshold dynamics method II: Applications to wetting dynamics. Journal of Scientific Computing, 81(3), 1860-1881, 2019.

[42] D. Wang, H. Li, X. Wei, and X. P. Wang, An efficient iterative thresholding method for image segmentation, J. Comput. Phys., 350, 657-667, 2017.

[43] D. Wang and B. Osting, A diffusion generated method for computing Dirichlet partitions, J. Comput. Appl. Math., 351, 302-316, 2019.

[44] D. Wang and X.P. Wang, The iterative convolution-thresholding method (ICTM) for image segmentation. arXiv preprint arXiv:1904.10917,2019.

[45] D. Wang, X. P. Wang, and X. Xu, An improved threshold dynamics method for wetting on rough surfaces, J. Comput. Phys., 392, 291-310, 2019.

[46] M. Wang, X. Wang, D. Guo, A level set method for structural topology optimization, Computer Methods in Applied Mechanics and Engineering 192, 227-246, 2003.

[47] N. Wiker, A. Klarbring, and T. Borrvall, Topology optimization of regions of Darcy and Stokes flow, Int. J. Numer. Meth. Engrg., 69, 1374-1404, 2007.

[48] Y. Xie, G. Steven, A simple evolutionary procedure for structural optimization. Computers \& Structures, Dec 3;49(5), 885-896,1993.

[49] X. Xu, D. Wang, and X. P. Wang, An efficient threshold dynamics method for wetting on rough surfaces, J. Comput. Phys., 330, 510-528, 2017.

[50] X. Xu, and W. Ying, An adaptive threshold dynamics method for three-dimensional wetting on rough surfaces. Communications in Computational Physics, 29(1), 57-79, 2021.

[51] T. Yamada, K. Izui, S. Nishiwaki, and A. Takezawa, A topology optimization method based on the level set method incorporating a fictitious interface energy, Comput. Methods Appl. Mech. Engrg., 199, 2876-2891, 2010.

[52] V. Young et al., 3D and multiple load case bi-directional evolutionary structural optimization (BESO). Structural Optimization 18.2-3, 183-192, 1999. 